\title{
Development and Construction of Large Size Signal Electrodes for the ATLAS Electromagnetic Calorimeter*
}

\author{
B. Aubert, J. Ballansat, J. Colas, C. Girard, \\ A. Jérémie, S. Jézéquel, J. Lesueur, G. Sauvage \\ Laboratoire de Physique de Particules (LAPP), IN2P3-CNRS, \\ F-74019 Annecy-le-Vieux Cedex, France. \\ D. Lissauer, D. Makowiecki, V. Radeka, S. Rescia, K. Wolniewicz \\ Brookhaven National Laboratory (BNL), Upton, NY 11973-5000, USA. \\ A. Belymam and A. Hoummada \\ Faculté des Sciences Ä̈n Chock, Casablanca, Morocco \\ A. Cherif, J.L. Chevalley, L. Hervas $\dagger$ C.P. Marin, P. Fassnacht, B. Szeless \\ European Laboratory for Particle Physics (CERN), CH-1211 Geneva 23, Switzerland. \\ J. Collot, M.L. Gallin-Martel, J.Y. Hostachy, P. Martin \\ Institut des Sciences Nucléaires, Université Joseph Fourier, IN2P3-CNRS, \\ F-38026 Grenoble, France. \\ M. Leltchouk, M. Seman \\ Nevis Laboratories, Columbia University, Irvington, NY 10533, USA. \\ P. Dargent, F. Djama, E. Monnier, C. Olivier, S. Tisserant \\ Centre de Physique des Particules de Marseille, Univ. Méditerranée, IN2P3-CNRS, \\ F-13288 Marseille, France. \\ G. Battistoni, L. Carminati, D. Cavalli, G. Costa, M.Delmastro, \\ M. Fanti, L. Mandelli, M. Mazzanti, L. Perini, F. Tartarelli \\ Dipartimento di Fisica dell'Universitá di Milano and INFN, I-20133 Milano, Italy. \\ E. Augé, W. Boniventoł D. Fournier, P. Puzo, L. Serin, C. de la Taille \\ Laboratoire de l'Accélérateur Linéaire, Université de Paris-Sud, IN2P3-CNRS, \\ F-91898 Orsay Cedex, France. \\ F. Astesan, B. Canton, D. Imbault, D. Lacour, F. Rossel, P. Schwemling \\ Laboratoire de Physique Nucléaire et de Hautes Energies, \\ Université Paris VI et VII, IN2P3-CNRS, F-75252 Paris Cedex 05 , France
}

11th May 2004

\begin{abstract}
We describe the electric circuits (electrodes) which polarize and read out the Lead-Liquid Argon electromagnetic calorimeter for the ATLAS detector. The paper covers design and material choices of the circuits as well as their production in industry. We also show how the electrodes integrate into the calorimeter and conclude with results from groups of electrodes making up calorimeter modules.
\end{abstract}

\footnotetext{
*Submitted to Nucl. Instrum. Methods A (2004)

${ }^{\dagger}$ Corresponding author: Luis. Hervas@cern.ch

${ }_{\ddagger}$ Now at INFN-Cagliari
} 


\section{Introduction}

ATLAS is one of the experiments being prepared for the LHC pp collider at CERN, Geneva. One of the main components in the ATLAS detector, the electromagnetic calorimeter, uses the liquid argon (LAr)lead sampling technique with accordion shaped geometry.

The description of the EM LAr calorimeter can be found elsewhere [1]. Here we just note that it is housed inside 3 cryostats, a barrel cryostat containing the Electromagnetic Barrel calorimeter (EMB), and two end-cap cryostats which in addition to the Electromagnetic End-Cap calorimeters (EMEC) hold the Hadronic End-Cap (HEC) and the Forward (FCAL) calorimeters.

The stack making up the EM LAr calorimeter is composed of three elements:

Absorbers The absorbers (made of lead glued between two thin stainless steel sheets) are $2.2 \mathrm{~mm}$ thick plates which are formed to accordion shape. The edges are glued to G10 bars for mechanical support.

Electrodes The readout circuits are made of 3 copper layers insulated by 2 layers of polyimide. This part is developed further in this paper.

Intergap spacers The intergap spacers made of honeycomb structured NOMEX ${ }^{\circledR 1}$ paper impregnated with phenolic resin, keep the electrodes centered in the gap between two absorbers.

Structural elements are needed to keep the absorbers in place. In the case of the barrel, where the G10 bars are in contact with each other and precisely define the LAr gap, stainless steel rings at the outer and thin G10 rings at the inner calorimeter radii rigidify the cylinder. The end-cap geometry is given by rings (composite material or aluminum) with indexation holes which house pins locating the position of the absorber G10 bars.

The main difference between the barrel and end-cap is the gap geometry. In the barrel the LAr gap can be kept at constant (2.1 mm half-gap) thickness from front to back by opening the folds of the accordion. The HV value is the same for all gaps $(2 \mathrm{kV})$. The end-cap geometry opens the gap at increasing radius. For mechanical reasons, two concentric (a "big" outer and a "small" inner) wheels have half-gaps varying from 0.9 to $3.1 \mathrm{~mm}$. This requires varying HV to keep the detector response independent of $\eta$. In a practical way the HV has 9 steps from 0.9 to $2.5 \mathrm{kV}$.

The charge deposited in the LAr gaps is collected on the cells of readout circuits (electrodes) which at the same time are used to polarize the gap. Fig. 1 shows the basic calorimeter cell with its granularity. While the $\phi$ divisions are achieved by ganging together several electrodes, the $\eta$ granularity and the depth separations are made by subdividing (etching) the copper surface of the circuit.

For reasons discussed below, the detection volume is covered by circuits with rather large surface $\sim 2 \mathrm{~m}^{2}$, of four different types. Starting from flat boards made on flexible substrate, the circuits are formed to the accordion shape following the gap. They are three-layer copper-polyimide printed circuit boards (PCB). The two outer layers are connected to the HV, while the inner layer, connected to the readout channels, collects by capacitive coupling the current induced by charges drifting in the LAr gap. The cells of the outer layers are divided into pads separated by resistive coating to protect the preamplifiers from accidental HV sparking.

This paper describes design issues in chapter 2, both the geometrical layout as well as the material choices. In chapter 3 we show the production process of the electrodes in industry, including quality assurance and controlled parameters. Chapters 4 and 5 explain the preparation and stacking of electrodes into the calorimeter modules. Finally in Chapter 6 we cover some results obtained with the calorimeter modules. They directly relate to the quality and performance of the electrodes.

\section{Electrode Design}

Since the first prototypes of the accordion calorimeter [2], flexible circuits with three copper layers separated by two insulating layers have been used. At that time circuits were within "industry standard" dimensions $\sim 60 \times 60 \mathrm{~cm}^{2}$. However given the dimensions of the calorimeter, at least circuits of seven types would have been needed for covering the whole central rapidity, with anyway one of the dimensions exceeding $60 \mathrm{~cm}$. This segmentation would imply large mechanical precision mounting difficulties

\footnotetext{
${ }^{1}$ Nomex $^{\circledR}$ is a registered trademark of DuPont Company
} 


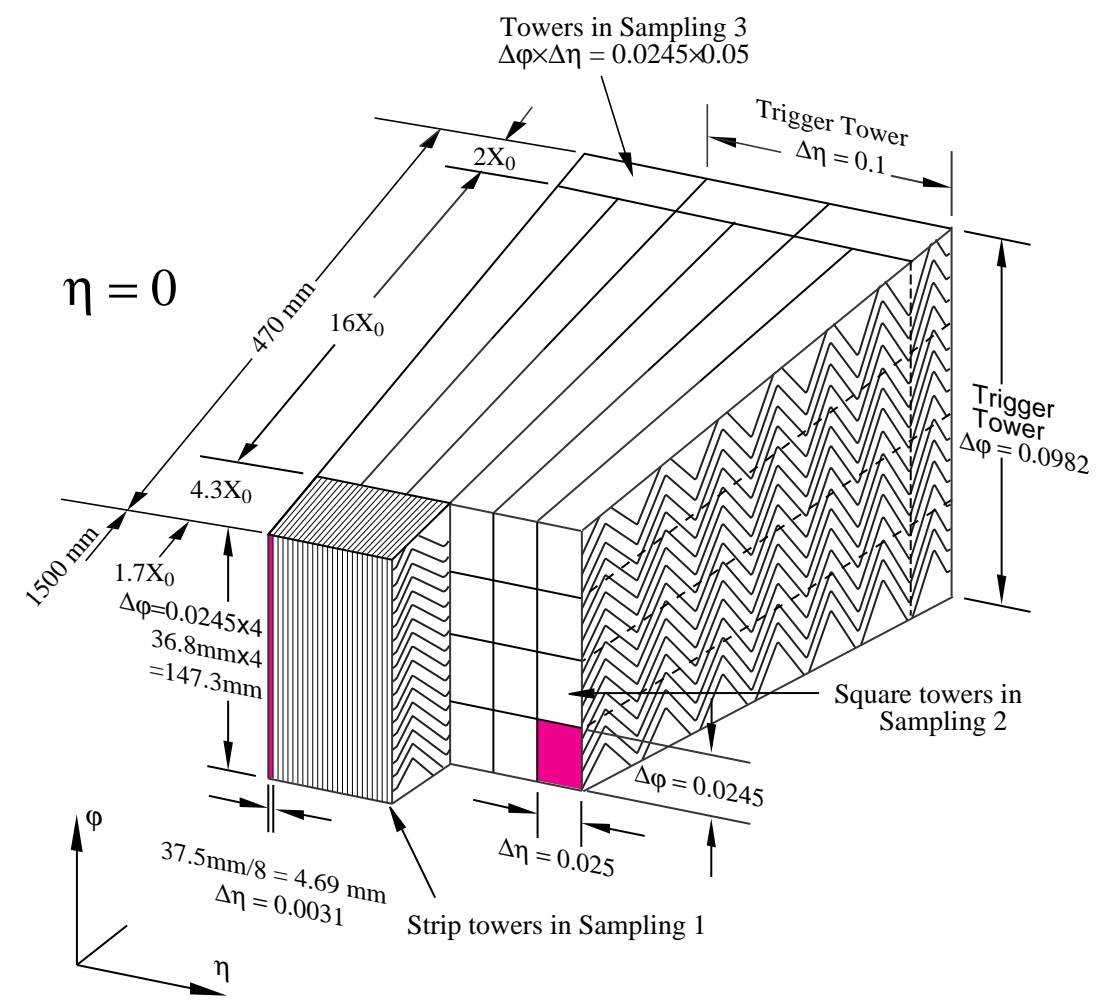

Figure 1: Schematic view of the typical calorimeter granularity with the divisions in $\eta$, $\phi$ and longitudinal depth.

and generate many dead areas without readout. Mainly these two reasons led to a development program where "large size" (up to $2 \mathrm{~m}^{2}$ ) circuits would be used, reducing the number to 2 types in the barrel and 2 types in the end-cap.

\subsection{Geometry}

In the following sections we discuss the geometrical choices leading to the design of the electrodes.

\subsubsection{Electrode types}

Fig. 2 shows a schematic overview of the EM calorimeter system and its integration in the ATLAS detector. The different pseudorapidity regions are shown.

The half-barrel region is around $3 \mathrm{~m}$ in length and $50 \mathrm{~cm}$ deep. This last dimension becomes $80 \mathrm{~cm}$ when the accordion is extended flat. While it might be possible to manufacture flexible circuits of $3 \times 1 \mathrm{~m}^{2}$, it would be rather difficult to manage all the production steps and subsequent handling. Thus it was decided to split the length in two circuits, further named types A and B, which thus become of $\sim 2 \mathrm{~m}^{2}$ area each.

Type A covers $0<\eta<0.8$ and type B covers $0.8<\eta<1.5$. Fig. 3 (upper) shows the external dimensions of the flat electrodes as well as the pattern etched on the signal layer. A gap of $1 \mathrm{~mm}$ is left between the two circuits at $\eta=0.8$. The barrel electrodes are fixed at their mid point onto the G10 bars of the absorbers by $5 \mathrm{~mm}$ pins. During the cool down of the calorimeter a contraction (relative to the absorbers) of $\sim 0.4 \mathrm{~mm}$ of the edges is expected. Thus the A-B gap would increase to $\sim 2 \mathrm{~mm}$.

The end-cap calorimeter is made of two concentric, inner and outer wheels. Each one is covered by one electrode, types D (outer wheel) and C (inner wheel).

The overall dimensions of flat $C$ and $D$ electrodes are shown in fig. 3 (lower). This design has been made given the desired acceptance coverage $(1.375<\eta<2.5$ and $2.5<\eta<3.2$, for D and C respectively), the position of the active volume along the beam in ATLAS, and its depth, the folding geometry (radius of curvature and variation of the folding angle with radius), and various routing, connection and mechanics constraints [3]. 


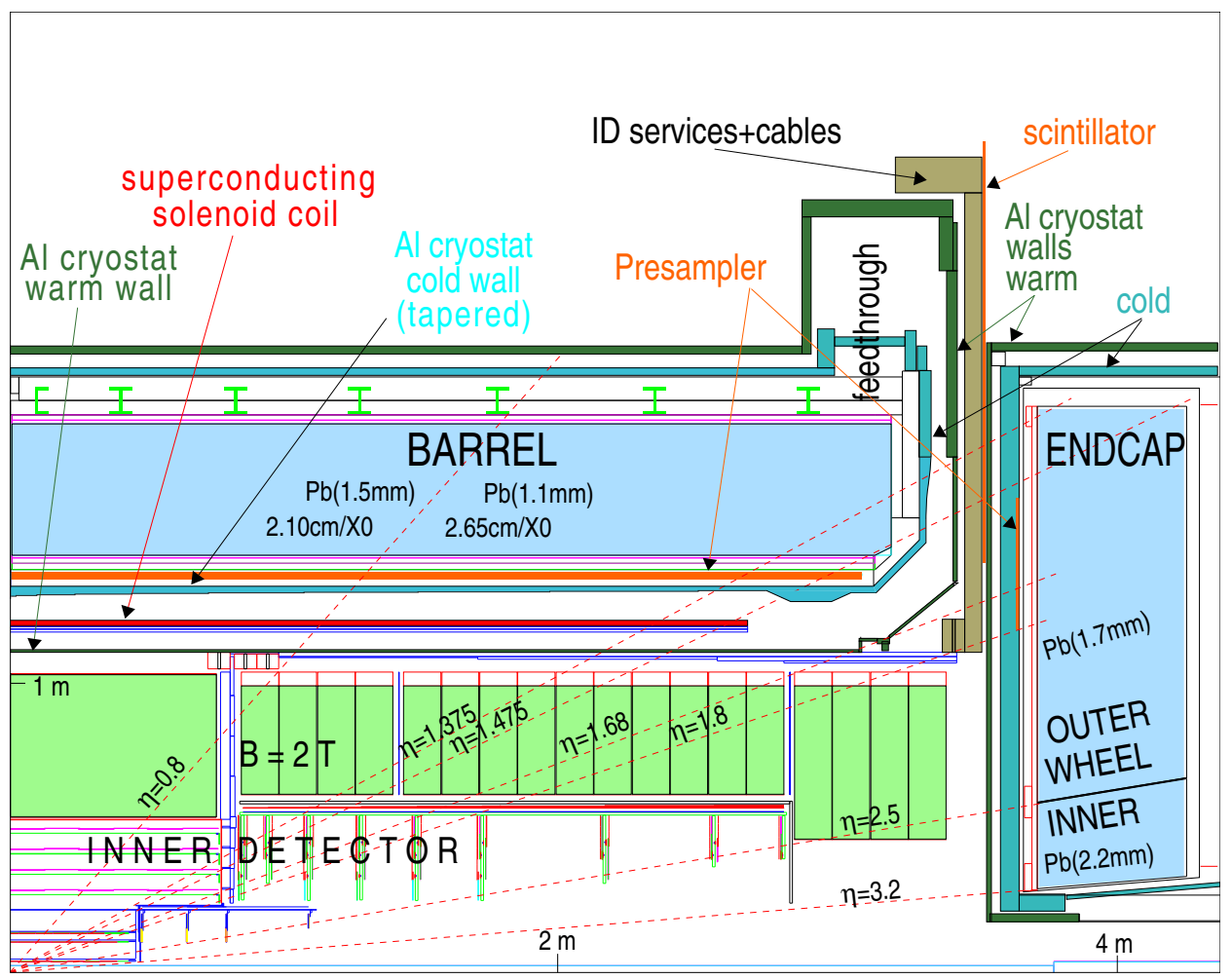

Figure 2: A quadrant section of the electromagnetic calorimeter and its surroundings in ATLAS. Note the Barrel half cylinder and the end-cap outer and inner wheels. Lines of pseudorapidity mark borders relevant for the electrode design.
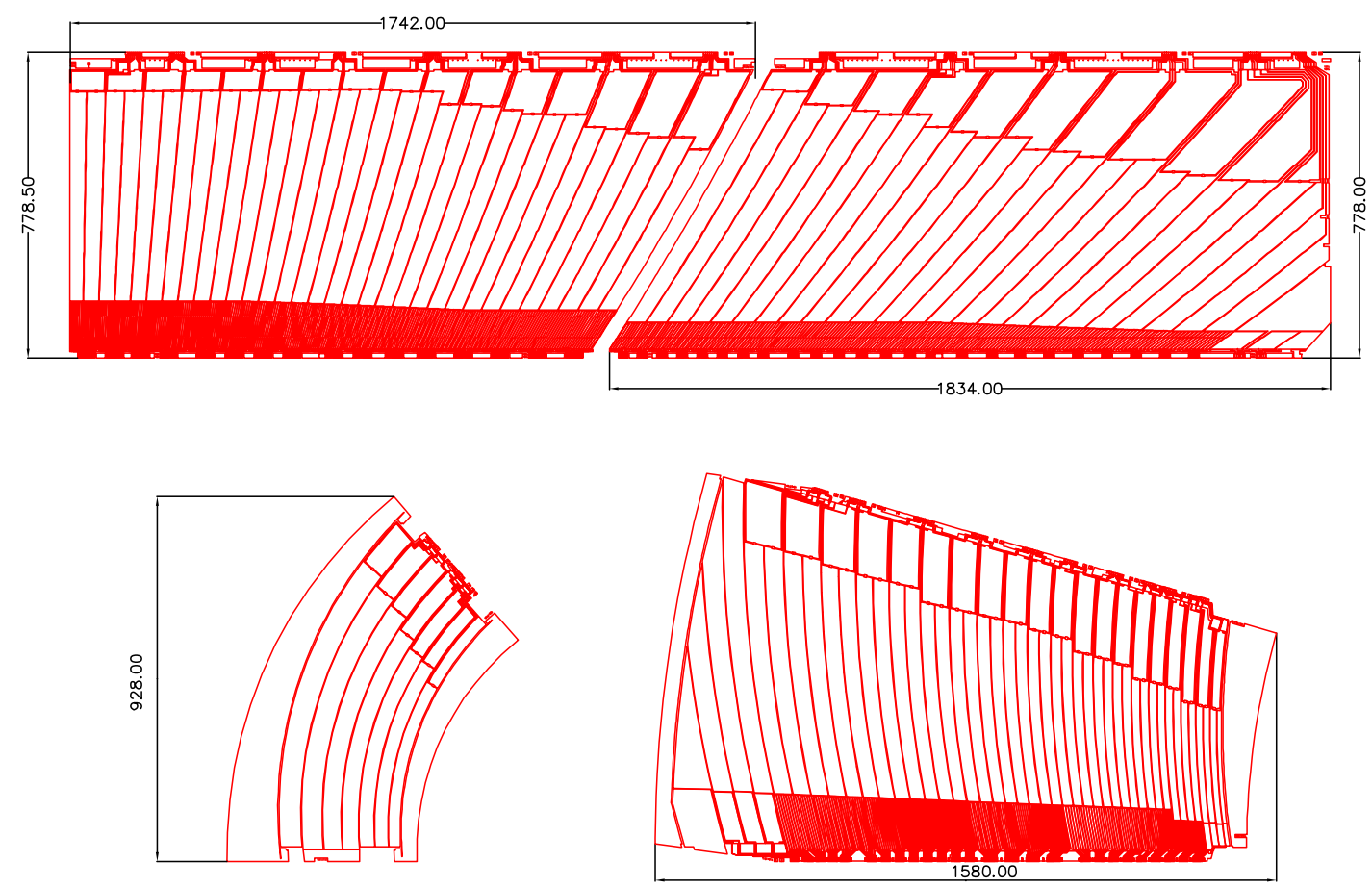

Figure 3: Layout of the signal layer for the four different types of electrodes: A, B (barrel), C, D (end-cap) at flat. Dimensions are in mm. Drawing are at equal scale. 


\begin{tabular}{|c|c||c|c|c|}
\hline Electrode & $\eta$ range & Front & Middle & Back \\
\hline \hline $\mathrm{A}$ & {$[0-0.8]$} & $\sim 0.003 \times 0.1$ & $0.025 \times 0.025$ & $0.05 \times 0.025$ \\
\hline \multirow{3}{*}{$\mathrm{B}$} & {$[0.8-1.350]$} & $\sim 0.003 \times 0.1$ & $0.025 \times 0.025$ & $0.05 \times 0.025$ \\
& {$[1.350-1.400]$} & $\sim 0.003 \times 0.1$ & $0.025 \times 0.025$ & - \\
& {$[1.400-1.475]$} & $0.025 \times 0.1$ & $0.075 \times 0.025$ & - \\
\hline & {$[1.375-1.425]$} & $0.050 \times 0.1$ & $0.050 \times 0.025$ & - \\
& {$[1.425-1.5]$} & $0.025 \times 0.1$ & $0.025 \times 0.025$ & - \\
& {$[1.5-1.8]$} & $\sim 0.003 \times 0.1$ & $0.025 \times 0.025$ & $0.050 \times 0.025$ \\
$\mathrm{D}$ & {$[1.8-2.0]$} & $\sim 0.004 \times 0.1$ & $0.025 \times 0.025$ & $0.050 \times 0.025$ \\
& {$[2.0-2.4]$} & $\sim 0.006 \times 0.1$ & $0.025 \times 0.025$ & $0.050 \times 0.025$ \\
& {$[2.4-2.5]$} & $0.025 \times 0.1$ & $0.025 \times 0.025$ & $0.050 \times 0.025$ \\
\hline $\mathrm{C}$ & {$[2.5-3.2]$} & - & $0.1 \times 0.1$ & $0.1 \times 0.1$ \\
\hline
\end{tabular}

Table 1: Granularity in units of $\Delta \eta \times \Delta \phi$ of the calorimeter cells

All the requirements from physics described below are given at liquid argon temperature. A thermal contraction of $3.6 \mathrm{~mm} / \mathrm{m}(300 \mathrm{~K}$ to $90 \mathrm{~K})$ has been used, to draw electrodes at room temperature.

\subsubsection{Samplings and Granularity}

Physics considerations drive the division of the detecting volume, and thus of the pattern on the electrode surface into several longitudinal samplings and cells. Each sampling is etched into projective cells along the $\eta$ direction. The three longitudinal samplings (see fig. 1) have the following characteristics:

- The first sampling (front, S1), optimized for the $\gamma / \pi^{0}$ separation, ends at $6 X_{0}$ including $\sim 1.6 X_{0}$ of dead matter in front of the active part of the calorimeter; the granularity is $\Delta \eta=0.025 / 8$.

- The second sampling (middle, S2) from 6 to $24 X_{0}$, has been optimized to contain photon showers up to $50 \mathrm{GeV}$ and has a granularity of $\Delta \eta=0.025$.

- The third sampling (back, S3) covers the remaining depth $\geq 2 X_{0}$ and has a granularity of $\Delta \eta=0.050$ to help reconstruct high energy showers and discern between hadronic and electromagnetic showers.

As a principle these divisions and segmentation hold over the whole $\eta$ range thus over the four electrode types. However some exceptions had to be implemented to adapt to the particular geometry :

- Close to $\eta=0$, the total depth is $24 X_{0}$; in order to leave a minimum of $2 X_{0}$ in the third sampling, the second one is reduced to $22 X_{0}$.

- Electrode $C$ has only two samplings, separated after $24 X_{0}$. In this area precision of physics will be dominated by other factors, thus we chose to reduce the number of readout channels.

- For intermediate regions $(1.375<\eta<1.5$ and $2.4<\eta<2.5)$, where the amount of dead material has its maximum, the $\mathrm{S} 1$ granularity is reduced to $\Delta \eta=0.025$. In the $1.375<\eta<1.5$ region S3 is merged with S2.

- For $1.8<\eta<2.4$, the S1 strip pitch would be below $5 \mathrm{~mm}$ if the cell size is kept at $\Delta \eta=0.025 / 8$, which is difficult to achieve, and would result in large cross-talk between strips. The strip granularity has been optimized to keep a minimal pitch of $5 \mathrm{~mm}$. A coarser granularity $(\Delta \eta=0.100)$ is used for both samplings of electrode C.

- Theoretical separations between samplings would cross folds, and thus, have to be shifted in order to ensure a minimum distance of $5 \mathrm{~mm}$ between the fold curvature edge, and the resistive ink edge (see section 2.1.4).

The final granularity is summarized in table 1 . 

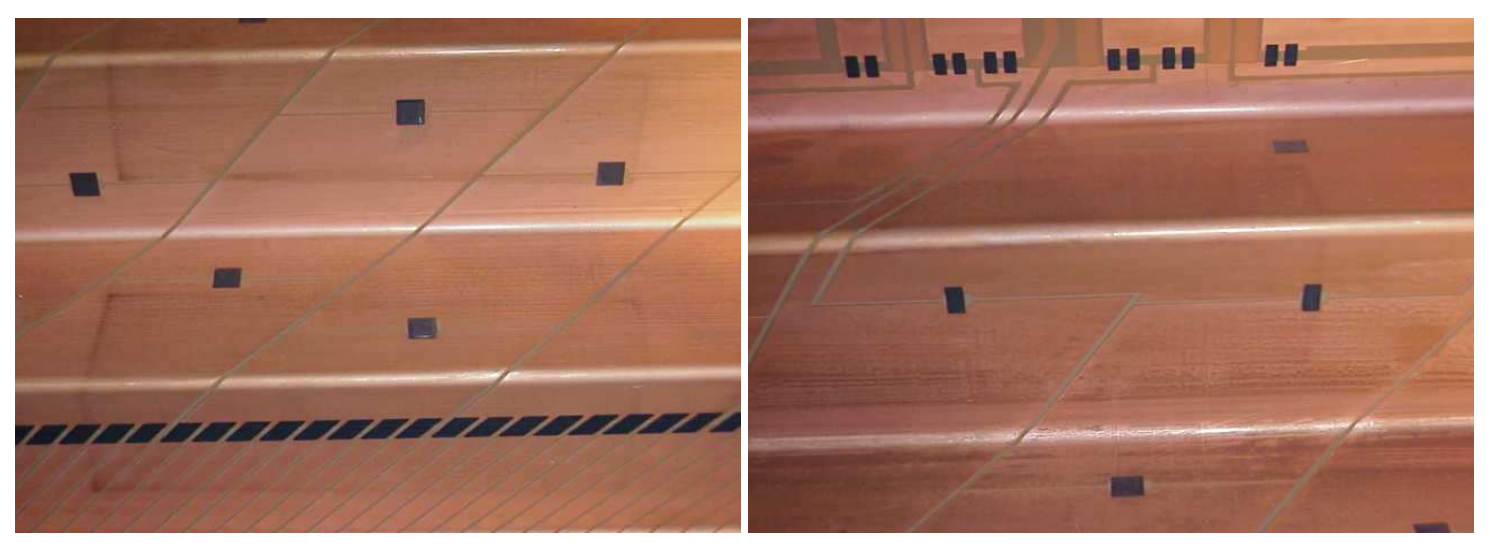

Figure 4: Left: Strips-to-middle resistors (lower part of the picture) connecting eight strips to each middle cell. Also shown are resistors dividing the middle cells in smaller pads. Right: Pair of high-voltage distribution resistors (upper) connecting the $\mathrm{HV}$ bus to the back sampling or directly to the middle cells by narrow copper traces. Both pictures are from a bent B electrode but representative of all other types.

\subsubsection{Electrical separations, connection paths}

The full copper surface is divided into cells by etching away thin separations. This is done not only on the signal layer for the readout cells, but also on the two outer layers connected to high voltage. The electrical separation between HV cells was chosen as $1 \mathrm{~mm}$ between middle or back cells and $0.5 \mathrm{~mm}$ between front cells. This separation ensures that when in LAr, one cell's HV can be turned off (in case of emergency operation) while the neighboring is at full polarization. The pattern of the underlying signal cells is separated additionally by $0.5 \mathrm{~mm}$ to avoid capacitive crosstalk due to possible misalignment between layers.

The connection of the strips to the outer connectors is direct to the front connectors for front cells and to the back connectors for the back cells. These strip lines are typically $1 \mathrm{~mm}$ wide since they have short $(\mathrm{few} \mathrm{cm})$ paths. The middle cells connections exit to the back connectors and have rather long $(\sim 20 \mathrm{~cm})$ paths. Thus they are of larger width $(\sim 3 \mathrm{~mm}$ ) which has to be increased when the paths are longer (at higher $\eta$ ) to try to keep the line inductance constant over the cells.

Cell separation gaps can be seen in fig. 4 (left) for front and middle cells. Fig. 4 (right) shows separations between back cells as well as connection strips towards the connectors.

\subsubsection{Resistor choices, locations}

The distribution of the HV to the outer layers of the electrodes is realized by means of resistive silkscreened pads of carbon-loaded epoxy ink. The layout of the resistor pads is shown in Fig. 5 for the four types of electrode. This layout was driven by the following criteria:

1. avoid cross-talk between calorimeter cells

2. protect the input of the preamplifiers

3. minimize dead regions in the front sampling

4. have some redundancy where possible

Other criteria are dictated by mechanical reasons mainly due to the geometry of the accordionshaped electrodes. Indeed this geometry excluded the possibility of a resistor coating spread uniformly on the whole electrode surface. Moreover, once the discrete resistor solution has been chosen one has to be careful to:

1. keep the safest possible distance from the bending region to minimize risks of resistor breakage

2. avoid alignment of resistors along the $z$-coordinate (r-direction) of the barrel (end-cap) electrodes which would create a line depleted in copper. Resistors on such a line have shown a higher probability of being damaged during bending. 

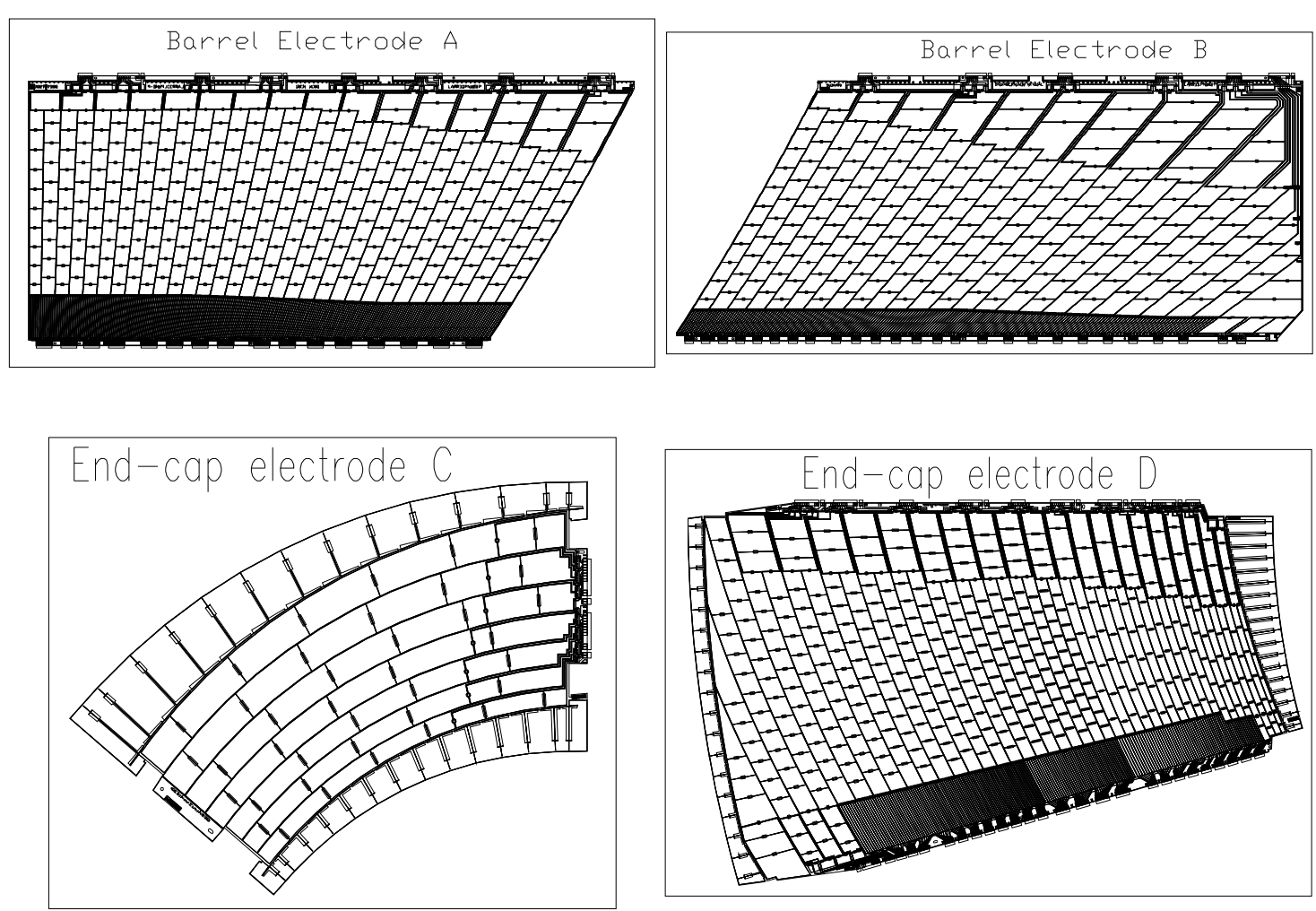

Figure 5: Layout of the resistors on the HV layer for the four different types of electrodes.

The HV of the whole surface is sectioned in $\Delta \eta=0.2$ areas each supplied by different HV sources. In order to provide some redundancy, the two HV layers, HV1 and HV2 have also separate sources. The $\mathrm{HV}$ is distributed from the back connectors to the cells via a bus of $6 \mathrm{~mm}$ width which connects the pads via silk-screened resistors.

The resistor layout can be briefly summarized in the following way (see Fig. 4). A series of resistors connects the back and middle sampling pads to the HV bus, located on the back side of the electrodes. Each strip of the front sampling gets the high voltage from the middle sampling to which it is connected through a resistor. The HV copper pads in the middle and back samplings are fragmented in smaller pads and interconnected by means of resistors. This geometry reduces the consequences on the preamplifiers of discharges. To reduce the risk of having unbiased regions as a consequence of a resistor failure, each connection from the HV bus to the middle and to the back samplings is made by two independent resistors in parallel. In the front sampling this is not possible due to space limitations. Additional safety is provided by resistors connecting the last pad of the middle sampling to the first pad of the back sampling. With this configuration, in case of failure of the direct connection from the HV bias line to the middle (back), the middle (back) sampling would be biased through the back (middle) sampling. Bringing the HV from the back of the electrodes minimizes dead regions in the front sampling and simplifies the HV cable distribution external to the electrodes.

To achieve the required precision on the energy measurement the resistance values must lie in a well-defined although rather wide range [4]. With the chosen resistive ink (see section 2.2.2) different nominal resistor values can be obtained by changing the aspect ratio of the rectangular resistor pads on the ink "layer" design.

The resistance lower limits are defined by the breakdown current of the input stage of the preamplifiers and by the maximum crosstalk acceptable between different samplings and between neighboring cells. For resistors connecting different samplings, a lower limit at $200 \mathrm{k} \Omega$ has been estimated. There can be resistances as low as $100 \mathrm{k} \Omega$ but their number should not exceed $1 \%$.

For resistors inside the middle and back samplings a safe limit that takes into account all the different cases is $10 \mathrm{k} \Omega$. 


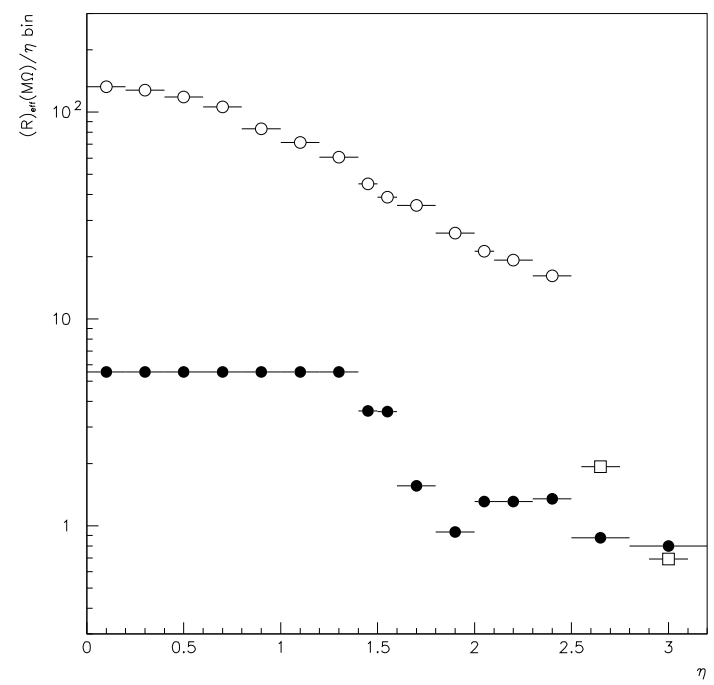

Figure 6: Total resistance from the strip to the high voltage line as a function of $\eta$ : maximum allowed (open symbols) and nominal value (filled circles).

The upper limits are defined by the maximum signal variation due to the voltage drop induced by the steady current coming from minimum bias events. A calculation was performed modelling the energy deposition of minimum bias events at high luminosity $\left(10^{34} \mathrm{~cm}^{-2} \mathrm{~s}^{-1}\right)$ in the calorimeter and imposing that the signal decrease does not exceed $10^{-3}$, matching our physics requirements. This corresponds to a maximum $0.3 \%$ drop in the biasing voltage.

A limit on the maximum acceptable value of the effective resistance measured from the strips to the high-voltage line was estimated [5] as shown in Fig. 6. From this, individual limits for each electrode resistor were deduced.

Resistor values are specially low for the end-cap cells since pile-up current increases swiftly with increasing $\eta$.

This explains the changes in dimension and number of resistors along $\eta$ for the $\mathrm{D}$ and $\mathrm{C}$ electrodes (larger pads with smaller aspect ratio and thus smaller resistances) while in the A and B electrodes resistor values do not vary (see fig. 5).

A maximum HV drop of 0.12 and $0.39 \%$ has been achieved in the end-cap outer and inner wheel, respectively. A safety factor of about 3 is kept in the outer wheel.

All these limits assume the nominal $1 \mathrm{M} \Omega / \square$ resistivity and a ratio $\mathrm{R}(\mathrm{T}=90 \mathrm{~K}) / \mathrm{R}(\mathrm{T}=300 \mathrm{~K})=3$ between the resistance value measured at liquid argon temperature and at ambient temperature (see section 3.3.3).

Table 2 summarizes the number of elements for each type of electrode.

\begin{tabular}{|c|c|c|c|c|}
\hline electrode type & A & B & D & C \\
\hline \hline Number of readout cells (Signal layer) & 303 & 230 & 280 & 14 \\
Number of HV pads (per face) & 580 & 427 & 677 & 53 \\
Number of resistors (for each face) & 660 & 485 & 775 & 74 \\
\hline
\end{tabular}

Table 2: Electrode geometry element counting for the four electrode types

\subsection{Material choices}

In this chapter we describe the characteristics of the materials used for the construction of the electrodes and of the components used to equip them (for test details see [6]). The electrodes consist of polyimide material, copper, glue and additional metallic and plastic elements used for electrical connections and mechanical support. All the materials have to fulfill a series of stringent requirements: 
1. they should be validated for operation at cryogenic temperature (the electrodes are immersed in liquid argon kept at about $89 \mathrm{~K}$ ) and should be able to withstand thermal constraints as the cryostat is cycled between ambient and operating temperature;

2. they should be rad-hard for the expected dose received by 10 years of LHC running $\left(10^{13}\right.$ to $10^{14} \mathrm{n} / \mathrm{cm}^{2}$ and about $\left.10^{4} \mathrm{~Gy}\right) ;$

3. have compatible thermal expansion coefficients among them. Moreover, the thermal expansion coefficient should be compatible with that of the lead-stainless steel absorbers which are interleaved between the electrodes in the detector;

4. they should not pollute the liquid argon neither in normal condition nor under the exposure to radiation [7];

5. it should be possible to bend the electrode at the desired radius of curvature without creating mechanical or electrical damage or delamination of the copper layer.

A big R\&D effort was pursued over several years in order to choose and validate all the electrode materials and possible industrial processing methods along these guidelines. The results are briefly summarized below.

\subsubsection{Laminates and glue}

The electrode boards consist of two main components, one double-sided and one single-sided copper clad polyimide sheets glued together, see fig. 7. The glue is thermosetting epoxy based. Other types of glue were evaluated (acrylic adhesives, UV-setting phenol adhesives) but they were discarded either because of a more delicate curing process or because they could not guarantee the same well-known radiation resistance of epoxy glues. To simplify the fabrication process, the glue is applied as a precured coating on the single-sided layer in a pre-polymerized stage (so called B-stage) directly by the laminating company. Once the copper sheets are processed (see section 3.1.1) the two polyimide sheets are pressed together and the glue curing is completed. The glue should also have a curing temperature compatible with the use of standard industry autoclaves or ovens.

The copper is of the HD (High Ductility-treated), ED (Electro-Deposited) type. This type of copper is safe for the electrodes to be bent without developing cracks. Due to the large width $(1 \mathrm{~m})$ of the boards it was not possible to use polyimide material laminated with RA (Rolled Annealed) copper.

Kapton ${ }^{\circledR 2}$ type E (a tetra polymer while standard Kapton is a two-component product) has been used as polyimide support because of its good mechanical properties. In particular, this type of material has the same thermal expansion coefficient as copper: this reduced the mechanical stresses of the electrodes due to large temperature changes. The laminate material used in the series production was manufactured by Arisawa Mfg. Co., Ltd. (Japan).

The thickness chosen for the circuit layers is shown in fig. 7. Polyimide of $50 \mu \mathrm{m}$ (rather than the more industry standard $35 \mu \mathrm{m}$ ) was considered "safer" for long term 2KV operation. Copper in $35 \mu \mathrm{m}$ quality is needed to correctly form the accordion folds. The thicker $(25 \mu \mathrm{m})$ B-stage inner glue layer was required to properly mould and glue around the etched relief to be sealed in the inner signal layer.

\subsubsection{Resistive Ink}

The characteristics of the ink used for the silk screened resistors where dictated by requirements on the resistor geometry, resistance ranges and location on the electrode.

In addition to the general requirements previously mentioned, the ink

- should have a resistivity making it possible to obtain the required resistance values with a small enough resistor size for all pads;

- should be of easy application;

- should have a curing temperature compatible with the laminate material;

- should be flexible enough to tolerate after curing, limited stresses during bending;

\footnotetext{
${ }^{2}$ Kapton ${ }^{\circledR}$ is a registered trademark of DuPont Company
} 


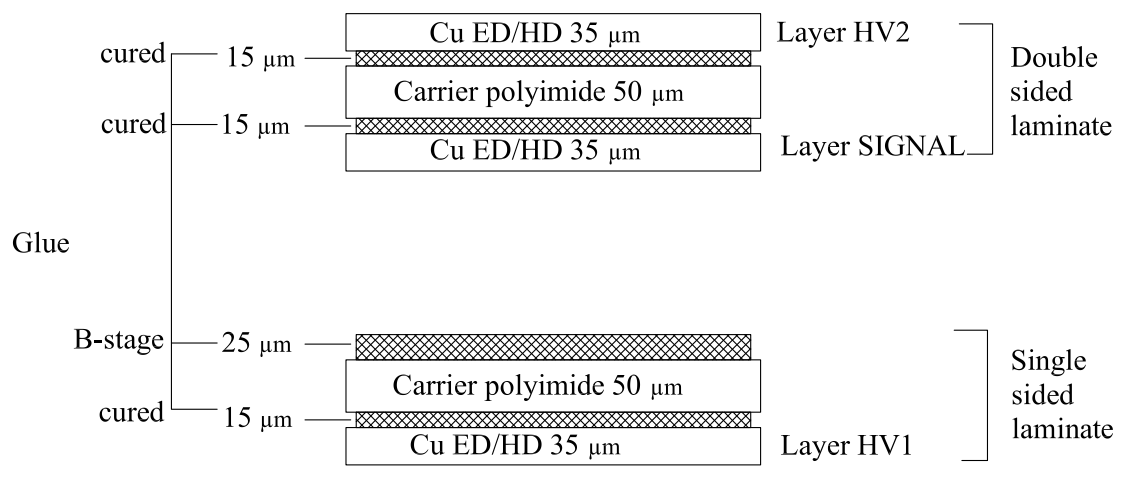

Total thickness $\sim 275 \mu \mathrm{m}$

Figure 7: Sketch of the laminate components making up the electrode sandwich

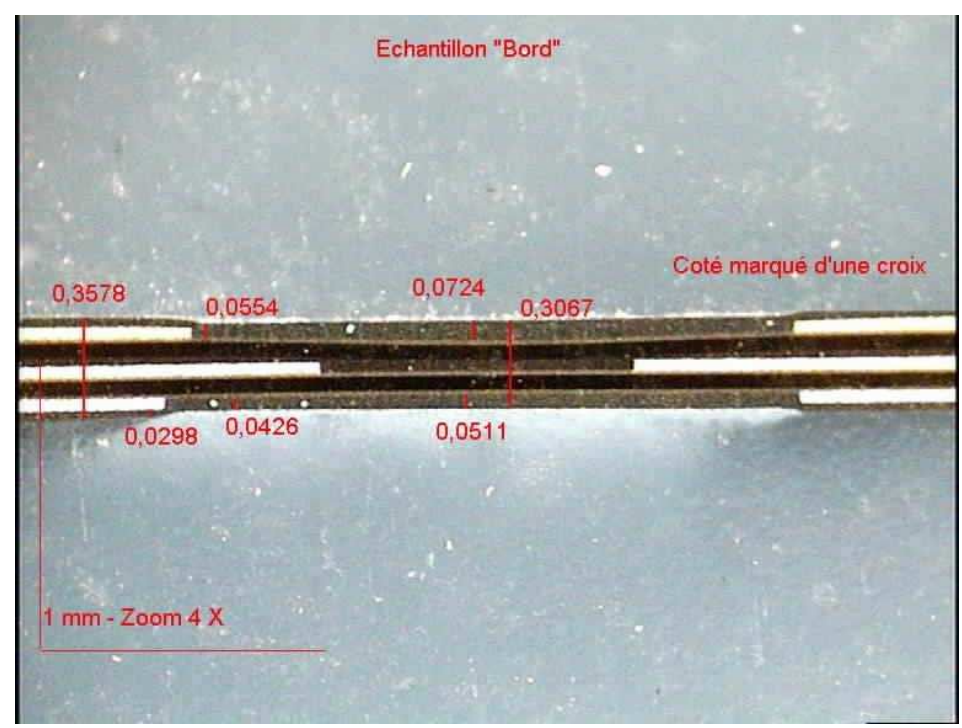

Figure 8: Silk screen resistor and substrate cross-cut. One can observe the 3 layers of copper (clear areas) interleaved with polyimide (black areas) and superposed by the ink (grey areas) on top and bottom sides, bridging the two copper pads by covering few $\mathrm{mm}^{2}$ of copper either side.

- should be compatible with the required operation at cryogenic temperature and its variation with the temperature should be limited and well controlled.

Several tests were made on reduced and real scale prototypes with different types of ink from various vendors having different ranges of resistivity, recommended thickness, curing cycles and number of passes. The final choice was the ink ESL ${ }^{3}$ D-RS 12116 (a one-component epoxy-based carbon loaded ink) with $1 \mathrm{M} \Omega / \square$ nominal resistivity for a $20 \mu \mathrm{m}$ thickness. A single-component epoxy was preferred because it permits longer pot lifetime and is known to be more rad-hard. The curing cycle suggested by the manufacturer is $170^{\circ} \mathrm{C}$ for two hours.

Radiation resistance and non-pollution have been assessed exposing electrode samples in a LAr bath to neutron and hadron beams [7].

Still even using the most flexible ink available, during the electrode mass production some brittleness of the resistive pads has been observed (see section 4.2.4). In fact the ink linking two copper layers is deposited on a surface with two steps of $35 \mu \mathrm{m}$ (the copper thickness) and these discontinuities are responsible for the dispersion in the resistance values and for the sensitivity to cracking specially after the bending phase of the electrodes. Fig. 8 shows a metallographic cut of a silk screen resistor. The different layers and the ink steps over the copper edges can be observed.

\footnotetext{
${ }^{3}$ Electro-Science Laboratory, Inc., King of Prussia, PA , U.S.A.
} 


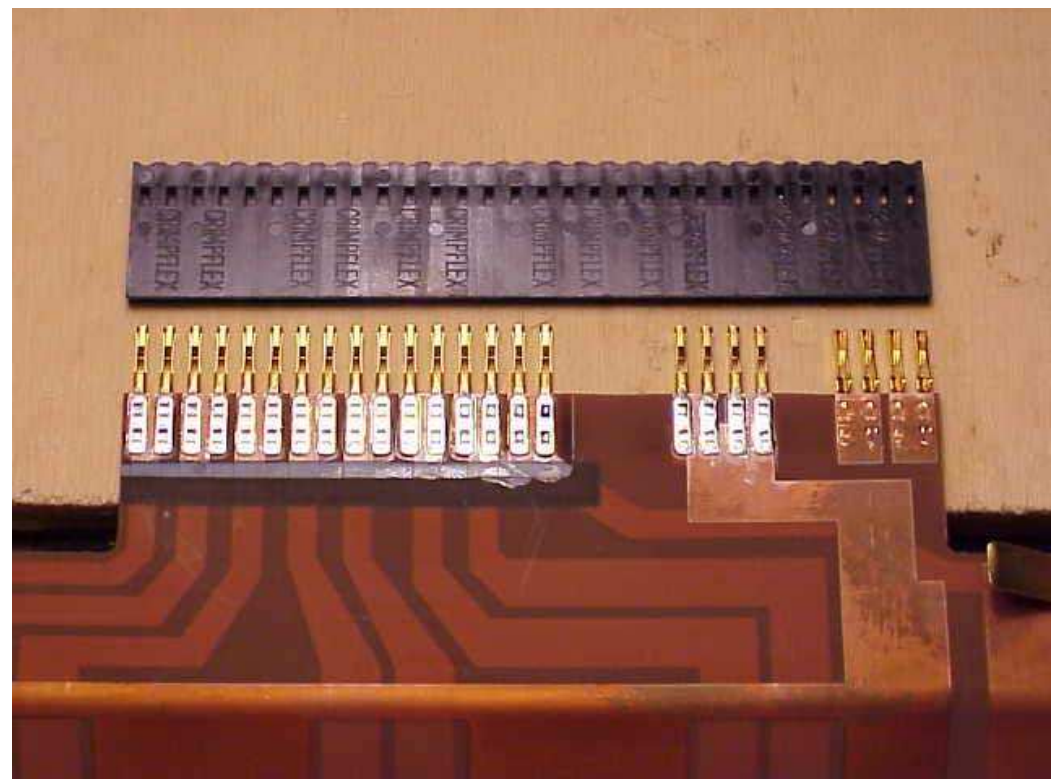

Figure 9: Naked connectors for high voltage lines (first eight pins from the right) and for signal extraction from middle and back. The first four contacts are upside down to connect to the bottom HV layer. Also shown detached is the black plastic housing

To limit the number of damaged resistors and limit the resistance dispersion various solutions were tried out. The density of the mesh of the silk screen was decreased from the nominal 200 lines per inch to 100 lines per inch giving in one inking pass a resistor thickness of $30 \mu \mathrm{m}$ with a better robustness. This, however, changed the mean resistivity to about $0.4 \mathrm{M} \Omega / \square$ instead of the nominal $1 \mathrm{M} \Omega / \square$ (see section 3.3.2).

\subsubsection{Connectors}

The electrical connections for the signals and high voltage must have modularity, high density, good reliability, radiation resistance and ease of installation on a flexible circuit. After the experience on early prototypes, the Nicomatic ${ }^{4}$ Crimpflex ${ }^{\circledR} 10025-32$ connectors have been chosen (see Fig. 9). These connectors are made of a row of female receptacles made of gold-plated phosphor bronze with a pitch of $2.54 \mathrm{~mm}$. They are crimped in 6 points to copper pads of the inner (outer) signal (high voltage) leads. The electro-mechanical contact is reinforced and secured by soldering. The insertion force is $1.5 \mathrm{~N} /$ pin, so $50 \mathrm{~N}$ are needed for one readout board row. A relatively high insertion force model has been chosen because it guarantees a higher retention for mechanical stresses like thermal cycles. The contact resistance is $6 \mathrm{~m} \Omega$. A protecting Ryton ${ }^{\circledR 5}$ housing (also from Nicomatic) is fitted on each group of connectors; the body also has the function of guiding the male connectors of the readout and HV boards. The required positioning precision along the electrode edge is $0.2 \mathrm{~mm}$ for compatibility with the G10 bars holding the front and back sides of the barrel electrodes.

For redundancy the signal leads of the middle and back samplings are connected with two pins in parallel; for each high-voltage connection four pins are used. A mock-up with about one thousand connections was tested with several cryogenic cycles. Although the connectors are guaranteed by the vendors to work for a minimum temperature of $-55^{\circ} \mathrm{C}$, their reliability in our application was assessed down to the liquid argon temperature.

\footnotetext{
${ }^{4}$ Nicomatic, Bons-en-Chablais', France

${ }^{5}$ Ryton ${ }^{\circledR}$ is a registered trademark of Philips Chemical Company
} 


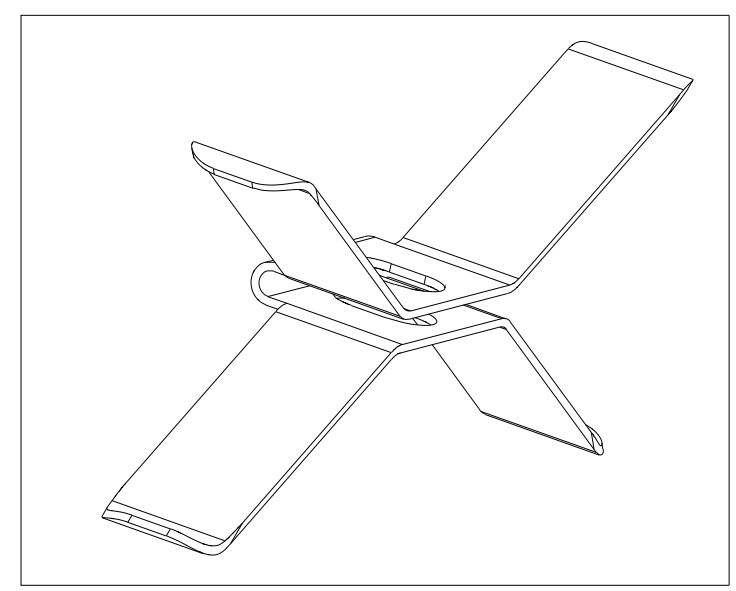

Figure 10: Barrel Ground springs

\subsubsection{Ground contacts}

In the barrel calorimeter, in the front and in the back side of the electrodes, gold plated, copper-beryllium, springs ${ }^{6}$ provide a ground return connection between the electrode and the absorber.

The springs have a shape with "four arms" (see fig. 10) to ensure stable mechanics and good electrical contact between the two absorbers and the electrode sandwiched between them. The springs are of two different sizes (the larger being located on the back side of the electrodes) and are soldered straddling the electrode on a specially designed copper pad.

With the chosen material thickness $(0.2 \mathrm{~mm})$ and geometry, a strength of about 235 (270) gr has been measured in working conditions (when the electrode is stacked in a module) for the small (large) springs in agreement with the expectations. In a test bench at warm and at cold (in a bath of liquid $\mathrm{N}_{2}$ ) the ohmic resistance of the ground contact has been measured to be about $50 \mathrm{~m} \Omega$. In the front side at $\eta=0$ and at $\eta=0.8$, where electrodes A and B are adjacent, a "half spring" is used due to the lack of space (i. e. a pair of spring arms is cut).

In the end-cap calorimeter the gap between the electrodes and the absorber is changing (between 1.5 and $7 \mathrm{~mm}$ at the chosen locations for the contacts) with $\eta$ (or radially from the beam pipe). Thus implementing the barrel concept would require sets of springs with different heights. This is why a fundamentally different approach was chosen. Instead of using a small surface with a strong force, we decided to contact a large surface by a small force. The contact is made by a small single-sided (gold plated) copper-polyimide sheet (called "flap") which touches the absorber on a given area while it is soldered to the ground pads on the electrode. The flap ${ }^{7}$ is held against the absorber by the spring effect of a pure polyimide tube which is compressed across its diameter. The tube ${ }^{8}$ is made of several interweaved layers of glued polyimide bands.

We chose tubes of seven different diameters $(2-6 \mathrm{~mm})$ which are only slightly compressed (fraction of a $\mathrm{mm}$ ) at the side where the gap is thinner and almost without any compression where the gap is larger. In this way we ensure that the "spring" tube remains in elastic regime. Tubes and flaps are glued together and the sets are soldered onto each side of the electrode.

The copper on the flaps is etched so that the contact part is full copper, separated from the edge by a guard ring. Thin paths carry the contact to the soldering point on the electrode. This prevents a full copper surface directly above signal paths which could cause large signal crosstalk.

With these contact surfaces and spring forces we measure typically contact resistances below $100 \mathrm{~m} \Omega$ both in air and in liquid nitrogen. Fig. 11 shows a flap-tube system on an electrode edge.

We note here how important it is to have sufficient and uniformly distributed ground contacts close to the paths of the readout signals. This fact could be well seen in module 0 electrodes where every second connector on the back of the electrodes had only one ground contact (instead of two), with connecting traces on one edge of the connector [8]. The inadequate ground contact topology causes an

\footnotetext{
${ }^{6}$ manufactured by Molmar, Milano, Italy

${ }^{7}$ made at Leoniflex, Burnhaupt le Haut, France

${ }^{8}$ manufactured by Polifibra SPA, Milano, Italy
} 

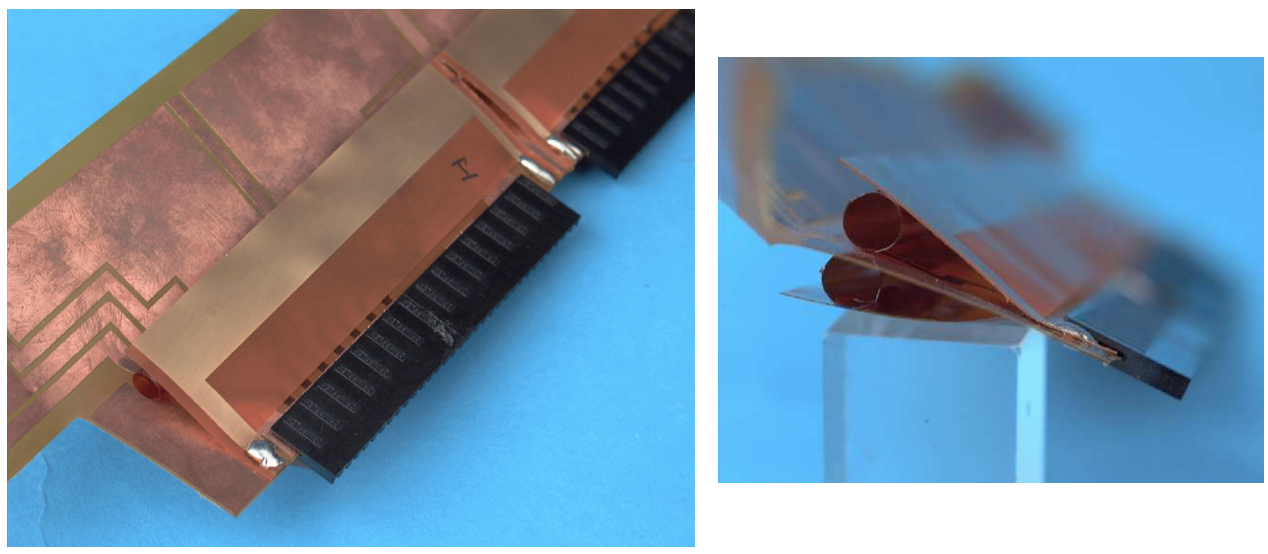

Figure 11: End-cap ground contact system. Note the "flaps" around the connector, their soldering points to the electrodes and the tubes to make the "spring" effect

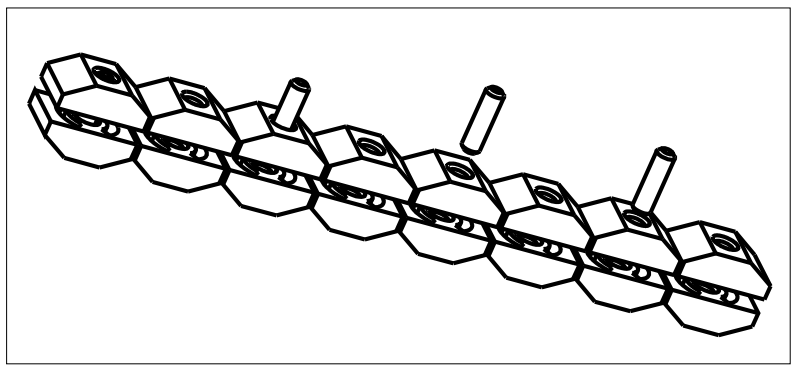

Figure 12: Barrel spacers

increase in the effective inductance of the associated signal paths which manifests itself as increased crosstalk between cells and signal shape deformation leading eventually (if not corrected by software) to a signal non-uniformity. For series production electrodes, the missing contacts were added into the circuit design.

\subsubsection{Spacers}

In the back side of the barrel electrodes, insulating rigid elements are mounted along the edge (see Fig. 12). Their purpose is to maintain the right geometry near the electrodes last bend when the electrode is inserted between two absorbers in the module stack. These elements ${ }^{9}$, called "spacers", are made of a PPF charged polymer, which has properties very similar to those of Ryton ${ }^{\circledR}$. To reduce cost and simplify the production, spacers of eight pierced elements are produced and then cut at different lengths. A pair of spacers of a given length is aligned with a set of holes on the electrode surface on both HV sides of the electrode and then tightened with conical pins (of the same material).

The shape of the spacers has been optimized to make it possible to mount them very close to the ground springs and prevent interference with the G10 bars of the absorbers once mounted in a module.

\footnotetext{
${ }^{9}$ manufactured by Politech, Carsoli(AQ), Italy
} 


\section{Electrode production}

The total amount of electrodes needed to equip the calorimeters is 6144 (2048 A, 2048 B, 1536 D, 512 C). They were all manufactured in industry ${ }^{10}$ during a two and a half year period. This production took place after extensive production developments for prototype electrodes and electrodes for prototype modules [8], [9], which allowed us to converge towards an optimally suited production process.

The process is based on chemical etching of the $\mathrm{Cu}$ layers to implement the cell segmentation and the connection fan-out structure. Other required steps are layer surface bonding, resistive ink serigraphy, circuit cutting out, connector crimping and soldering.

\subsection{Process description}

A flowchart of the production process can be followed in fig. 13. In the following we discuss some major steps and choices in this process.

\subsubsection{Key points in process}

Signal etching In order to get the best dimensional accuracy, the signal layer is first etched on the double sided laminate. With this, the other face of the double sided laminate is still full copper preventing large deformations due to heat or humidity treatments on the polyimide.

Layer bonding The autoclave gluing is a delicate step. We need to bond two square meter surfaces with no delamination or creases. A key difficulty is to remove any gas between the surfaces to prevent it from generating trapped "bubbles". This is achieved by preparing a sandwich of the laminates and wrapping them with:

- layers of materials which allow gas outflow

- a moulding layer which compensates for thickness differences on the surfaces to glue (e.g. on the etched separation lines)

- rigid steel surfaces to transmit uniformly the pressure

- a sealed bag to pump out the air and gas contents of the sandwich while curing

The autoclave cycle basically is preceded by a preheating and pumping step where most of the air between layers is pumped out. During the curing time itself pumping is continuously performed to remove any possible glue outgassing. The pattern etched on the signal surface (inner layer in the sandwich) has "channels" which cover the whole surface ending at the edges of the sandwich. This helps greatly in evacuating any trapped (or inside generated) gases and prevents bubble formation.

Layer alignment One of the main requirements (mostly driven by channel crosstalk prevention and mechanical precision for outcuts) is a good alignment between images in superimposed layers. For this :

- Films are controlled and measured before being used in production, not only for their pattern and dimensions, but specifically for their agreement over layers.

- Great care is taken to fix the films to the laminate in a precise and reproducible way which allows for small deformations. Only one precise hole is centrally placed on the laminates. The others are oblong holes which define accurately a reference system, tolerating some isometric deformation of laminates without losing precision. The layers and films are then clamped together by precision pins through the holes.

- Moreover, the designed pattern allows for small shifts by making the HV layer patterns oversized $(0.25 \mathrm{~mm})$ compared to the ones on the inner Signal layer.

\footnotetext{
${ }^{10}$ Cicorel SA (La Chaux de Fonds), Boudry, Switzerland
} 


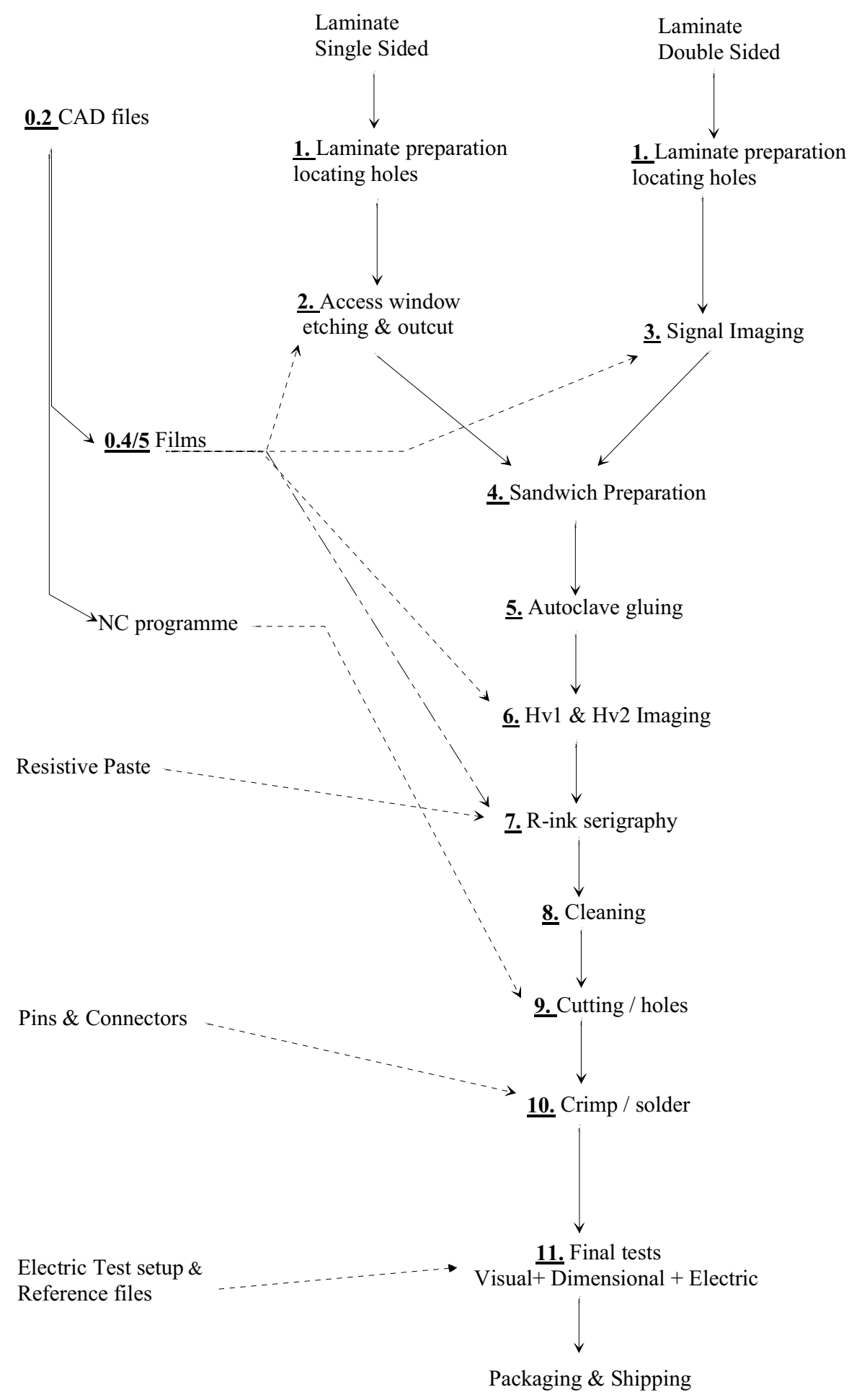

Figure 13: Electrode basic production steps flowchart 
Resistive ink serigraphy Tuning of the serigraphy process to obtain good values of the resistors presented several difficulties which had to be overcome.

As mentioned above a one component resistive paste on epoxy base was chosen to have reproducible values of resistivity The nominal resistivity value of a paste is given for "noble" substrate material typically ceramics or pure polyimide. However the resistors on the electrodes are generated by ink deposited on a polyimide surface which is covered by epoxy glue. Experimentally we discovered that the interaction of different laminate glues (from different vendors) with the same resistive ink, produces resistances which can differ by up to an order of magnitude. Also variations in the curing cycle produce analogous effects. Therefore we optimized a process which we followed for the production, where:

- one face of the electrode is inked with the appropriate mesh silk screen,

- the sheets are minimally dried,

- sheets are turned and the second face is inked and

- the sheets with ink on both sides are finally cured.

The final curing obtains good resistance values along with good mechanical properties. Reasonably flexible resistors are indeed needed to tolerate circuit handling and electrode bending into accordion form without breaking.

Final cutout After trying out several techniques, the Compute Numerically Controlled (CNC) machining with a $2 \mathrm{~mm}$ diameter mill cutter was identified as best suited. It mills from the edge the circuit sandwiched between two wood-cardboard martyr plates. A commercial multi-head CNC PCB machine was transformed in a large surface $\left(\sim 3 \mathrm{~m}^{2}\right)$ PCB mill.

In order to produce minimal metallic debris, the final cutout is all routed through polyimide-only areas except for the contact pin edges where we also have over a few $\mathrm{mm} 2$ layers of copper. The precision holes used to align the films to the circuits, are also used as reference to the CNC table.

\subsubsection{Process qualification}

For all the complicated electrode production processes, a contractual requirement of process qualification $(\mathrm{PQ})$ was imposed. For each fabrication step, PQ points were identified. Some examples are :

- Laminate cutting table

- Film : dimensional, superposition precision

- Vacuum pressed illumination table for photo-resist imaging process

- Autoclave sandwich preparation

- Serigraphy table positioning

\subsubsection{Quality Control $(\mathrm{QC})$ in process}

For a smooth and efficient production, points were identified as QC relevant and consequently QC actions were implemented as part of the process. A non-exhaustive list includes :

- Regular film control : dimensional and visual (dirt, scratches ...). Regular film replacement.

- Visual control of photoresist imaging. Manual re-touch.

- Etching time control and record

- Autoclave cycle parameters recording. Plate location inside autoclave.

- Regular peeling tests on glued laminate samples, for gluing cycle tracing

- Layer Staggering verification

- Recording of ink curing cycle parameters 


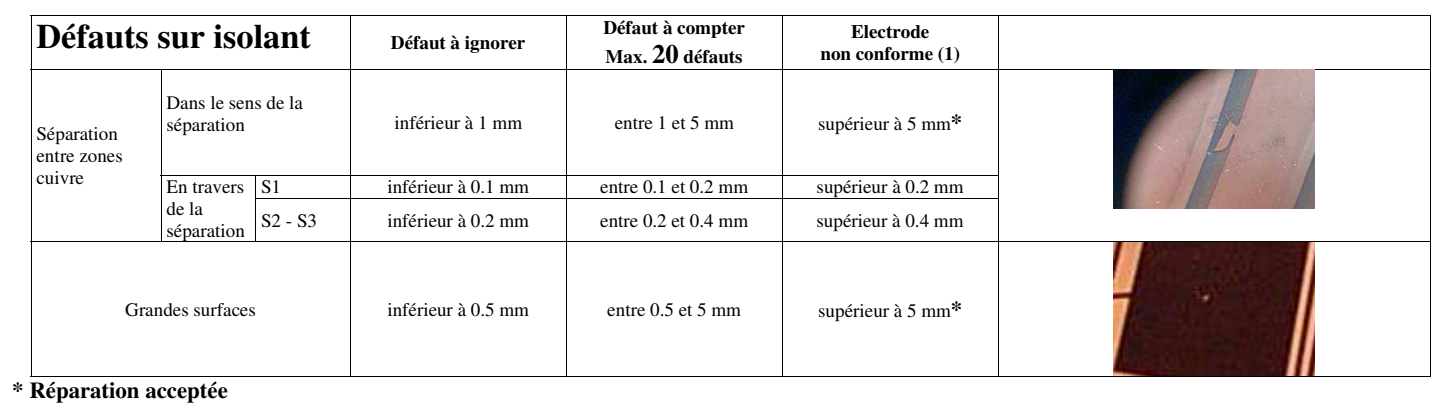

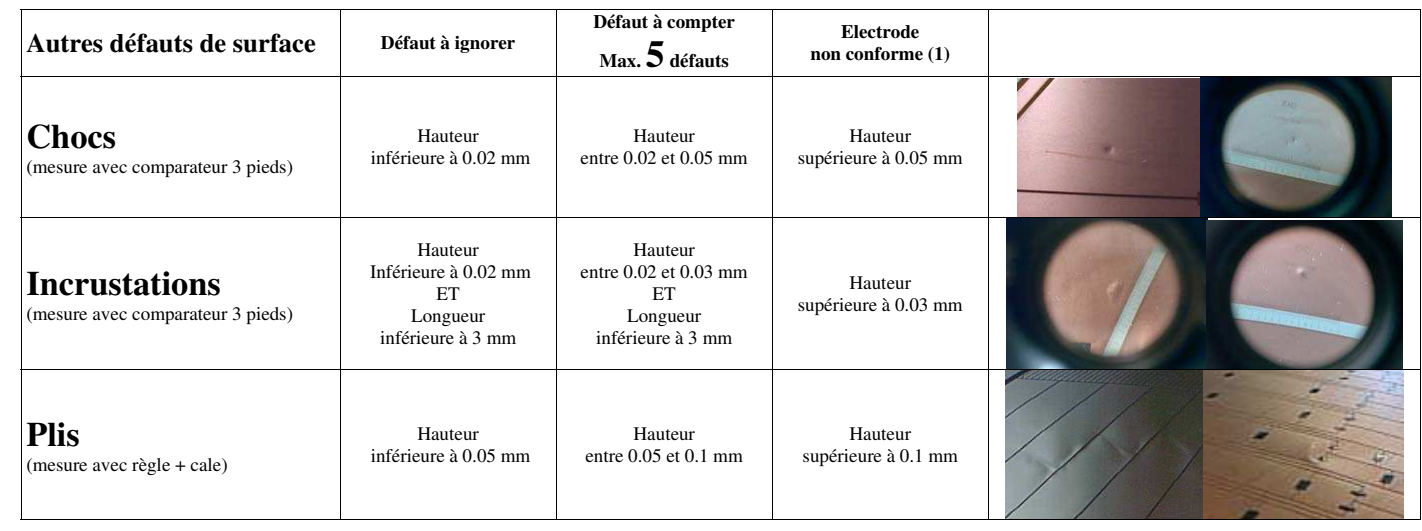

5

Figure 14: Example of visual inspection conditions document

\subsection{Production Quality Assurance (QA)}

A final QA control for each electrode was done according to a check list. The results were summarized in one control sheet per electrode to be filled by the operators. The main points are covered in the following sections.

\subsubsection{Visual inspection}

A document defining the acceptable defects for gluing, etching, cleanliness, handling, was worked out with the manufacturer. A sample of this is shown in fig. 14. For each electrode (and for each side) items covering those points were included in the QA list and had to be filled out by the operator. Electrodes not fulfilling the requirements were rejected. Electrodes with defects close to acceptance limits had to be examined by ATLAS collaboration personnel on an individual basis and in some cases electrodes were declared conform for delivery.

\subsubsection{Dimensional QA}

Dimensional measurements were performed on each electrode and recorded in the QA sheet. Key measurements were defined (e.g. hole diameter, edge-hole distances, image-edge distances, distances between optical targets which were specifically etched along with the rest of the circuit on the copper pattern for this purpose) and were required for each electrode. Also dedicated optical patterns which give a measure of the inter-layer superposition precision had to be inspected and recorded. More detailed dimensional measurements which are typically very reproducible (e.g. external dimensions, given by $\mathrm{CNC}$ machine precision) were additionally performed on a regular sampling of the produced electrodes. Fig. 15 shows a page of a guiding document with typical dimensional measurements to be performed on each electrode.

Fig. 16 shows the results of the metrology on one A electrode. Points are taken on the copper pattern, over the layers as well as in holes and cutouts. This precision metrology was performed regularly on delivered batches of electrodes after reception.

Over the electrode dimensions of $2000 \mathrm{~mm}$, typical results obtained along series production for some 


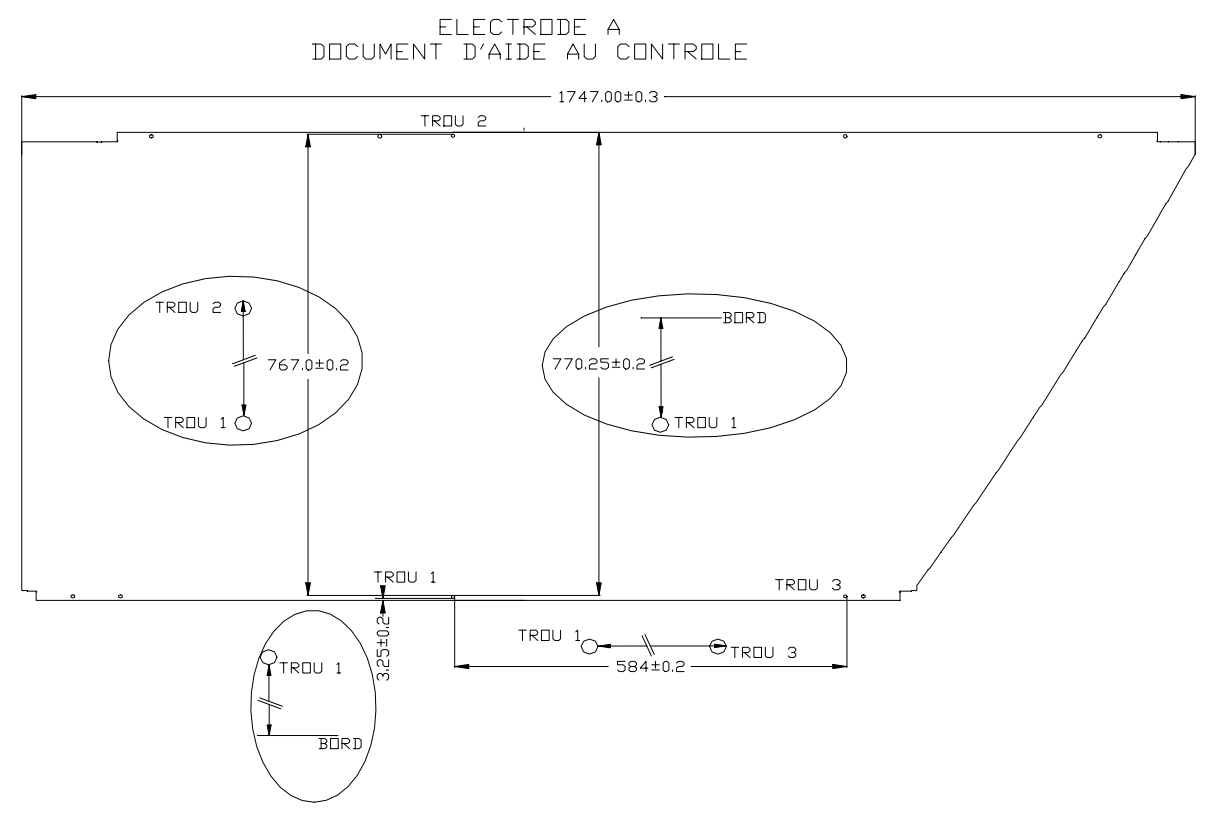

Figure 15: Guiding document for dimensional measurements

geometrical properties are shown in fig. 17. The distances shown are taken on the external cutout of the electrode and on the copper images on both electrode faces. The precisions obtained are at the level of a few $0.1 \mathrm{~mm}$.

Fig. 18 shows the image observed by transparency through a $\times 10$ magnifying glass of one superposition pattern. Two patterns were placed at each of the four corners of the electrode design. One pattern checks the relative displacement ( $x$ and $y$ combined) of the HV1 face (empty squares) relative to the signal layer (smaller inner squares), while the neighboring pattern (only partly seen in the photograph), records the displacement of the HV2 face also relative to the signal layer. The dimensions of the squares are etched such that the superposition precision can be read off (from 0.1 to $0.8 \mathrm{~mm}$ in $0.1 \mathrm{~mm}$ steps) directly by looking at the pattern through a magnifying glass and recording which one of the inner squares "touch" the external edge. While the required tolerance was better than $0.5 \mathrm{~mm}$ (given by the front cells separation, see section 2.1.3) the results obtained were typically between 0 and $0.2 \mathrm{~mm}$ for all 8 patterns on an electrode.

\subsubsection{Electrical QA: high-voltage tests}

When functioning in LAr, the two outer copper layers of the electrode are kept at high voltage while the inner layer, where the ionization signal is collected, is connected to the input of the preamplifiers (virtual ground). The operating high voltage is $+2000 \mathrm{~V}$ in the barrel ( $\mathrm{A}$ and $\mathrm{B}$ electrodes) and (due to the varying LAr gap) between $+900 \mathrm{~V}$ and $+2500 \mathrm{~V}$ in 9 discrete steps in the end-cap ( $\mathrm{C}$ and $\mathrm{D}$ electrodes). The high voltage test described in this section is meant to ensure that the electrode can hold these voltages without any appreciable leakage current (or even worse, discharge) between the inner and the outer layers. The high-voltage test was performed on each electrode. The test helped in identifying production problems and in rejecting defective electrodes. Due to its importance, the high-voltage test is also repeated (in a more sophisticated way) at the electrode test and assembly sites, after it has undergone further handling (bending, equipping) just before it is shipped to the module stacking sites (see section 4.2.2).

\section{Set-up}

The high-voltage test set-up consists of two cabinets (of eight shelves each), a multi-channel HV system (CAEN SY127 equipped with four A633 modules) with current trip capability and current monitoring with about $10 \mathrm{nA}$ resolution and an A303 CAEN interface for communicating with a computer. The data acquisition system is implemented in LabView on a PC running the Windows operating system. 

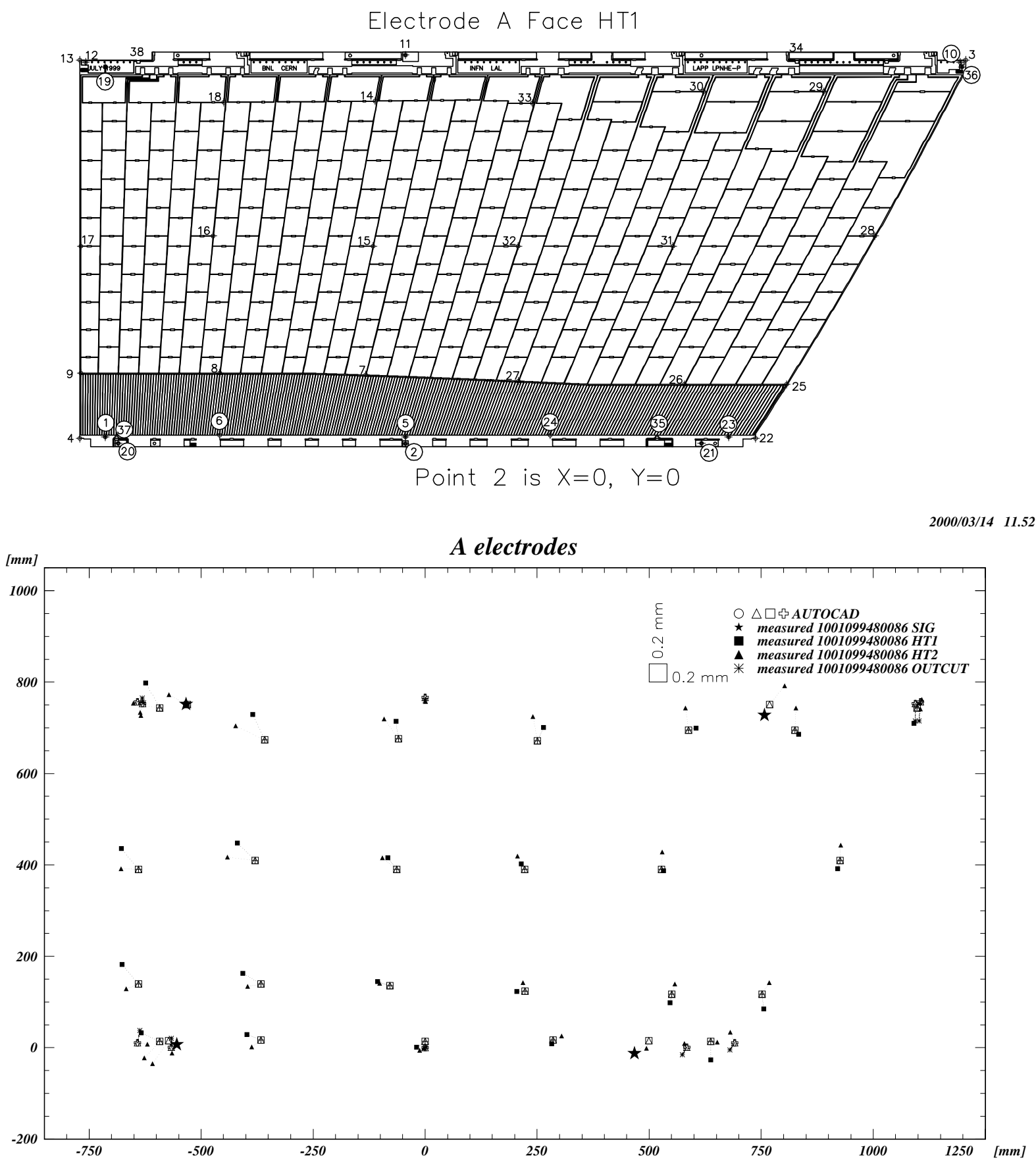

Figure 16: Dimensional electrode metrology. Reference points (Top) are taken over the outer high voltage layers (HT1, HT2) as well as in the inner signal (SIG) layer and the edges and holes (OUTCUT). Bottom, the various marks show the displacement between the nominal and measured positions expanded 100 times. 


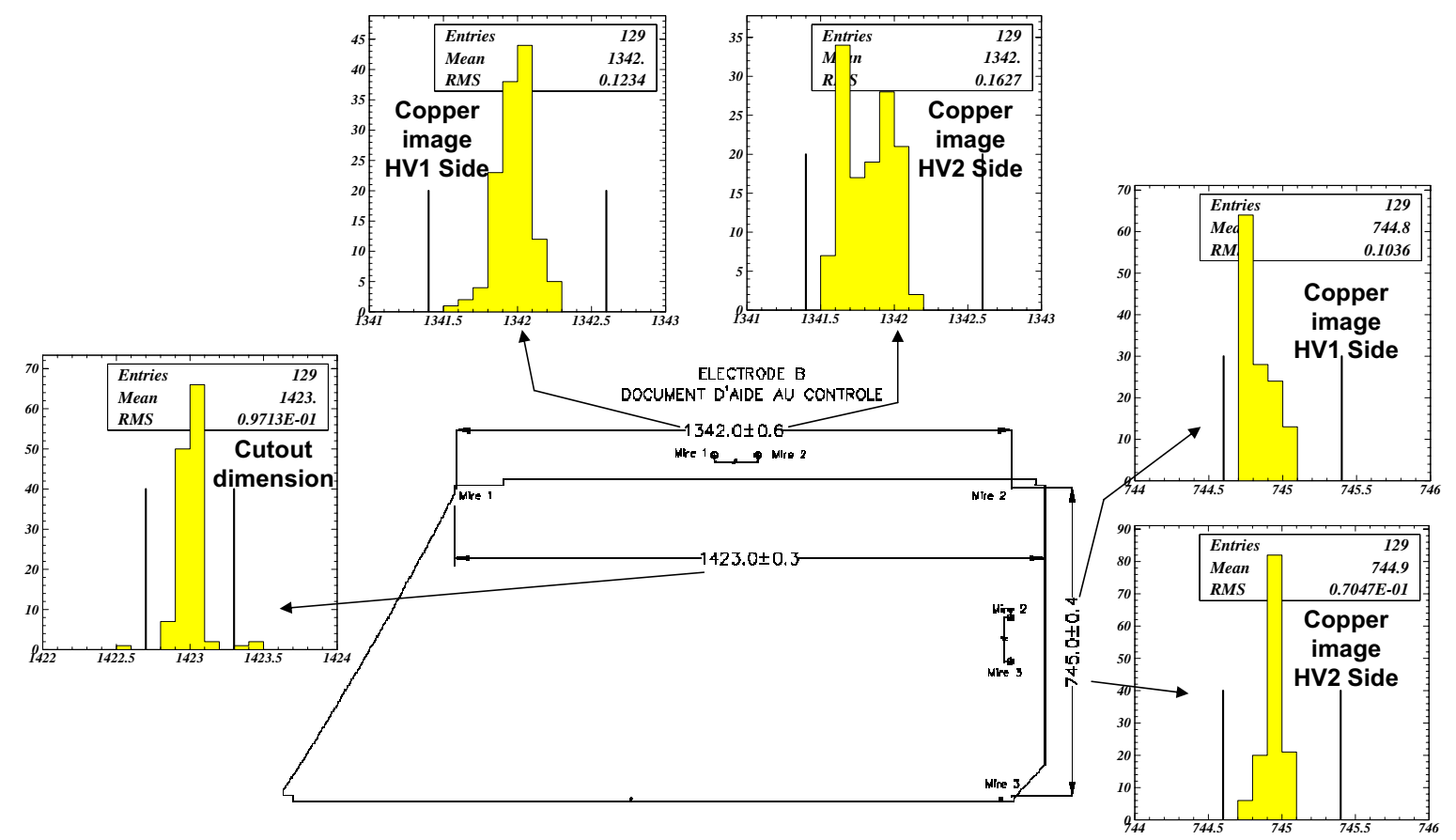

Figure 17: Statistical results of dimensional measurements on B electrodes over series production. Histograms show data for 129 B electrodes spread over the full series production during 2 years. Distances histrogrammed are on the external cutout (left) which show precisions better than $0.1 \mathrm{~mm}$ over $1.4 \mathrm{~m}$ and on copper etching (two distances on the two electrode faces) where the rms error 0.1-0.2 mm over $1.3 \mathrm{~m}$ is well within the tolerance required of $\pm 0.6 \mathrm{~mm}$.

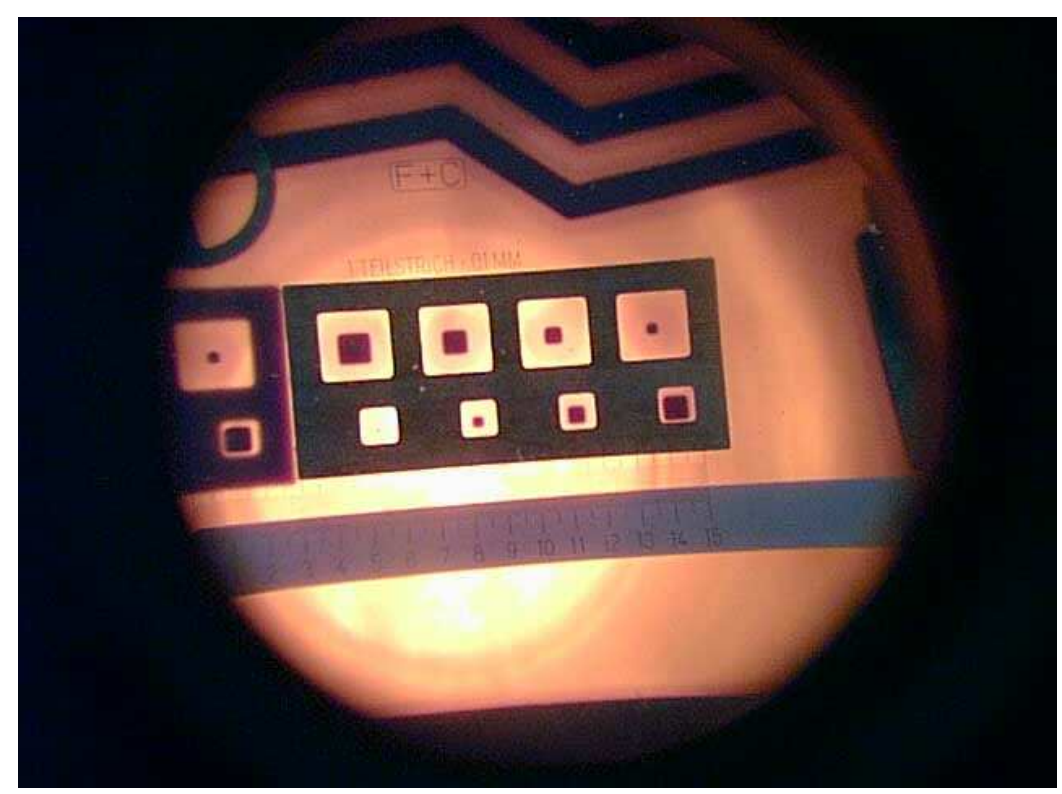

Figure 18: Superposition control pattern. While the squares seen inside the shape are copper on the inner (signal) layer, the outer shape is on the external (HV) layer. With a magnifying glass $(\times 10)$ and by transparency, seeing which square 'touches' the edges, one can read off directly (in $0.1 \mathrm{~mm}$ steps, from 0.1 -lower right square to $0.8 \mathrm{~mm}$-upper right square) the precision of the layer staggering. In this case the superposition would be better than $0.1 \mathrm{~mm}$. On each of the four corners, two patterns are used to verify the superposition of the external HV1 (HV2) layer to the inner signal layer. 

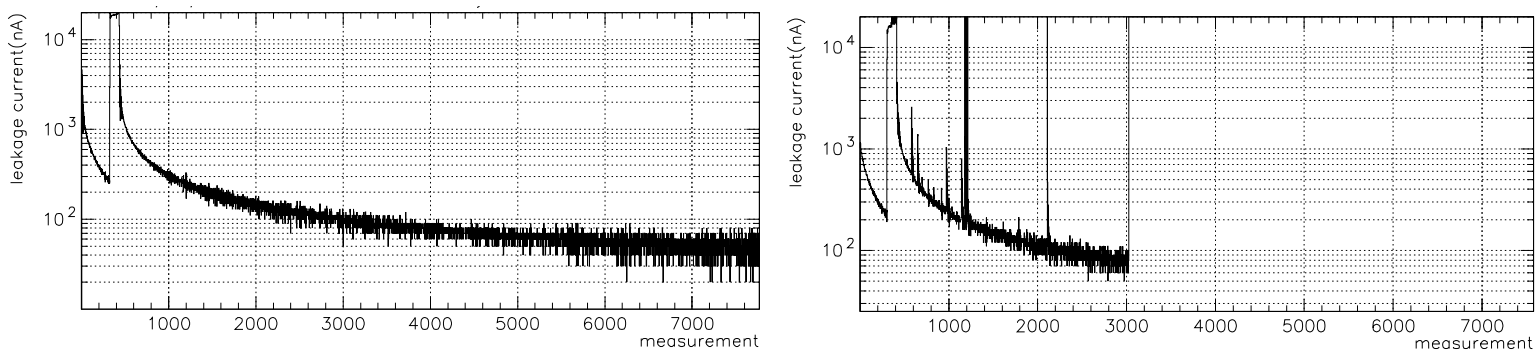

Figure 19: Leakage current as a function of time of a good electrode charged at $2000 \mathrm{~V}$ (left). The right plot shows the same quantities for a bad electrode. The current spikes in the end cause a trip of the test power supply. The horizontal scale corresponds to a total duration of one hour.

The system allows the testing of 16 electrodes of any type at the same time. The high voltage is applied to the two external electrode layers through the high-voltage connectors while the signal layer (through the strips, middle and back signal connectors) is kept to ground. The nominal test voltage is $-2000 \mathrm{~V}$. This voltage has been chosen to avoid discharges between adjacent copper traces kept at different potential on the electrode surface which have a minimum distance of $2 \mathrm{~mm}$. The plateau value is reached in two steps: a slow ramp to $-1000 \mathrm{~V}$ and, after a few minutes, a final ramp up to $-2000 \mathrm{~V}$. During this time the leakage current is sampled with a frequency of about $2 \mathrm{~Hz}$ and recorded for further analysis.

As the test is performed in air, it is important that the electrodes are properly cleaned and dried to avoid corona effects. The ambient humidity has to be controlled at all times and kept at a value less than $50 \%$ (relative humidity).

\section{Test Procedures at the Production Site}

The leakage current as a function of time for a good electrode is shown in Fig. 19. The leakage current is recorded starting after the first ramp at $-1000 \mathrm{~V}$. One can see the charging of the electrode up to the point where the voltage is brought to $-2000 \mathrm{~V}$. Then one observes the slow charge of the electrode determined by the large capacity value of the electrode. After a few hours the leakage current goes asymptotically to about $10 \mathrm{nA}$ which is the resolution of the measuring instruments. Higher currents would indicate a problem in the electrode.

As it was not practical, especially at the production site, to perform a test lasting several hours, waiting for the slow charge of the electrode to complete, it was decided to perform one hour long tests and to cut on the asymptotic leakage current at that time. From experience on early prototypes and on pre-production electrodes it was decided to flag as bad all electrodes having a leakage current above 300 nA after 1 hour. Similarly, as the charge of the electrode is usually pretty smooth, experience from early tests showed that having a r.m.s. of the last 100 measurements lying above $30 \mathrm{nA}$ might be an indication of problems. Results of measurements performed for all electrodes (all types combined) can be seen in fig. 20.

The test may fail either because by the end the current doesn't meet the above criteria or because during the test the leakage current is so high (e.g. due to a resistive bridge or a shortcut) that it causes a trip of the HV supply. Moreover, sparking on the electrode is not acceptable.

\section{Test results}

A total of 7352 (2450 A, 2443 B, 587 C and 1872 D) electrodes were tested. Of these, 56 electrodes (21 A, 22 $\mathrm{B}, 0 \mathrm{C}$ and $13 \mathrm{D}$ ) failed the test, a $0.76 \%$ percentage of the total. These numbers may include electrodes rejected for other reasons at a subsequent step of the production quality control. Similarly, electrodes rejected at an earlier step of the production chain are not included in these countings.

All electrodes failing the high-voltage test were carefully examined in order to trace and understand potential problems either in the design or in the production chain. In some cases the problem could be corrected and the electrode accepted.

Failures could be summarized in one of the following categories: 

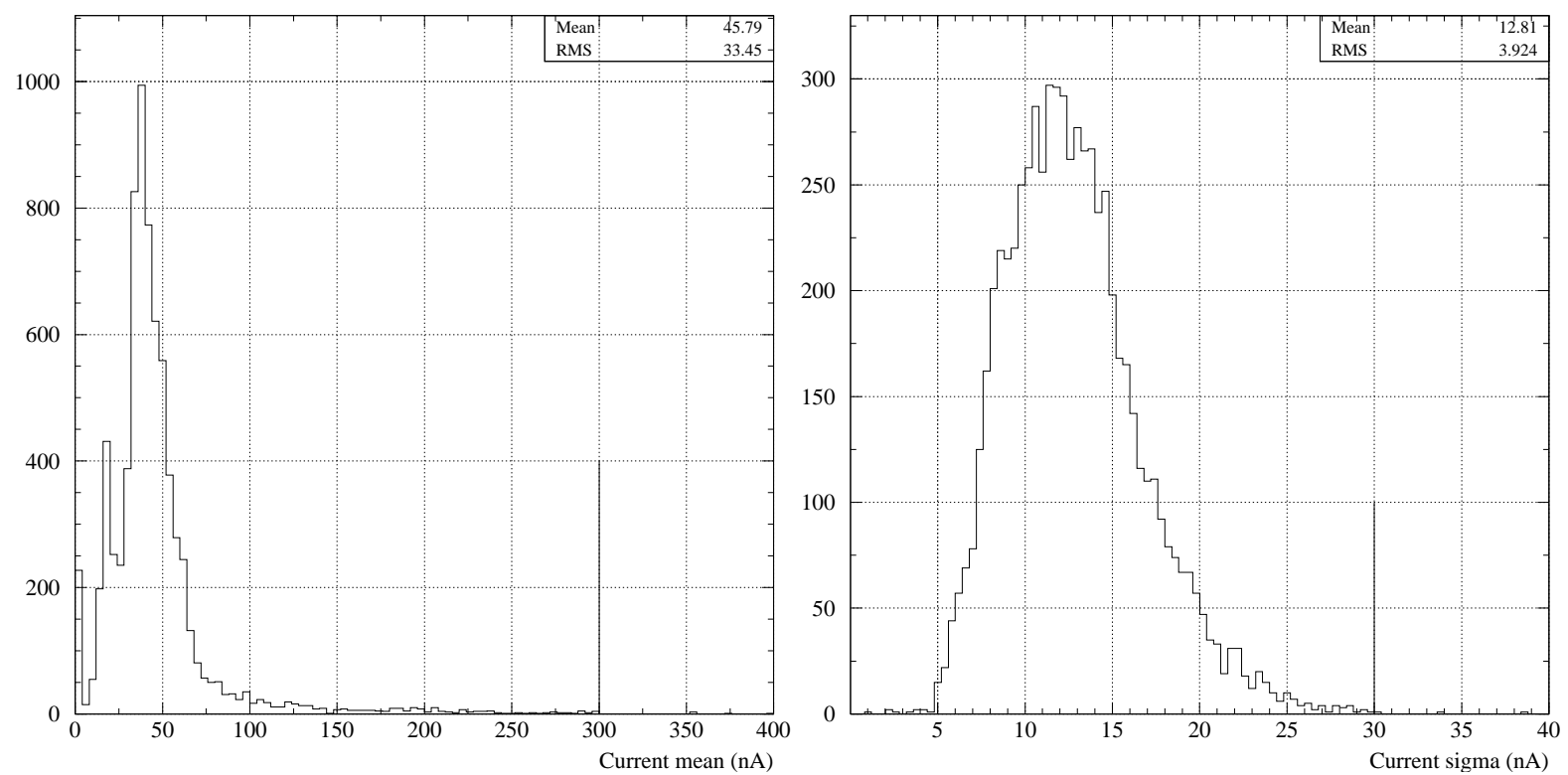

Figure 20: Average (left) and RMS (right) of the last 100 current measurements for all produced electrodes after 1 hour of running at $-2000 \mathrm{~V}$. The peaks at small currents in the average plots is due to the $\mathrm{C}$ electrodes which have smaller currents than A, B and D electrodes.

Inappropriate handling. During the production or testing process the electrode suffered a damage of the polyimide insulation between the HV and the inner copper layer. An example is shown in Fig. 21 (top left). In this case a "hole" was caused by a sharp object (probably the probes of a multi-meter used to check the resistive pads) which created a hole in the insulating layer. This type of problem disappeared rapidly after starting series production when more careful handling practices were implemented.

Bad copper etching. High-voltage problems may also be caused by bad etching of the HV copper layer (see Fig. 21, top right picture) which leaves a spurious copper trace which causes arcing between high voltage and ground pads. This problem can be easily fixed by chemical etching of the extra copper (see section 4.2.2).

Bad gluing. Bad gluing between the single-layer and the double-layer laminates at the edge of the electrode (see Fig. 21, bottom left picture) can also cause arcing.

Design issues. Some early problems of the electrode design were spotted thanks to the high-voltage test. The short horizontal dark line at the center of the fig. 21, bottom right, is a section of one signal lead which is connected to virtual ground. Under certain manufacturing circumstances, this part of the lead is not covered by isolating polyimide, as it should be, in order to guarantee a safety gap between high voltage and ground traces. For a first series of electrode (like the one shown in Fig. 21, bottom right), this problem has been corrected by shortening (by additional chemical etching) the two closest HV layer strips (as done for the strips in this figure).

Most of these problems could not have been spotted easily by eye.

\subsubsection{Electrical QA : RC testing}

On flat and bent electrodes, resistance and capacitance measurements have been performed, allowing us to detect the following problems :

- measurement of capacitances between the signal layer and the HV layers allow the detection of any interruption in the inner copper layer, in the signal fan-out and in the crimped connectors.

- resistance measurements allow to check electrical continuity, and to check the resistivity of the silk-screened resistances. 


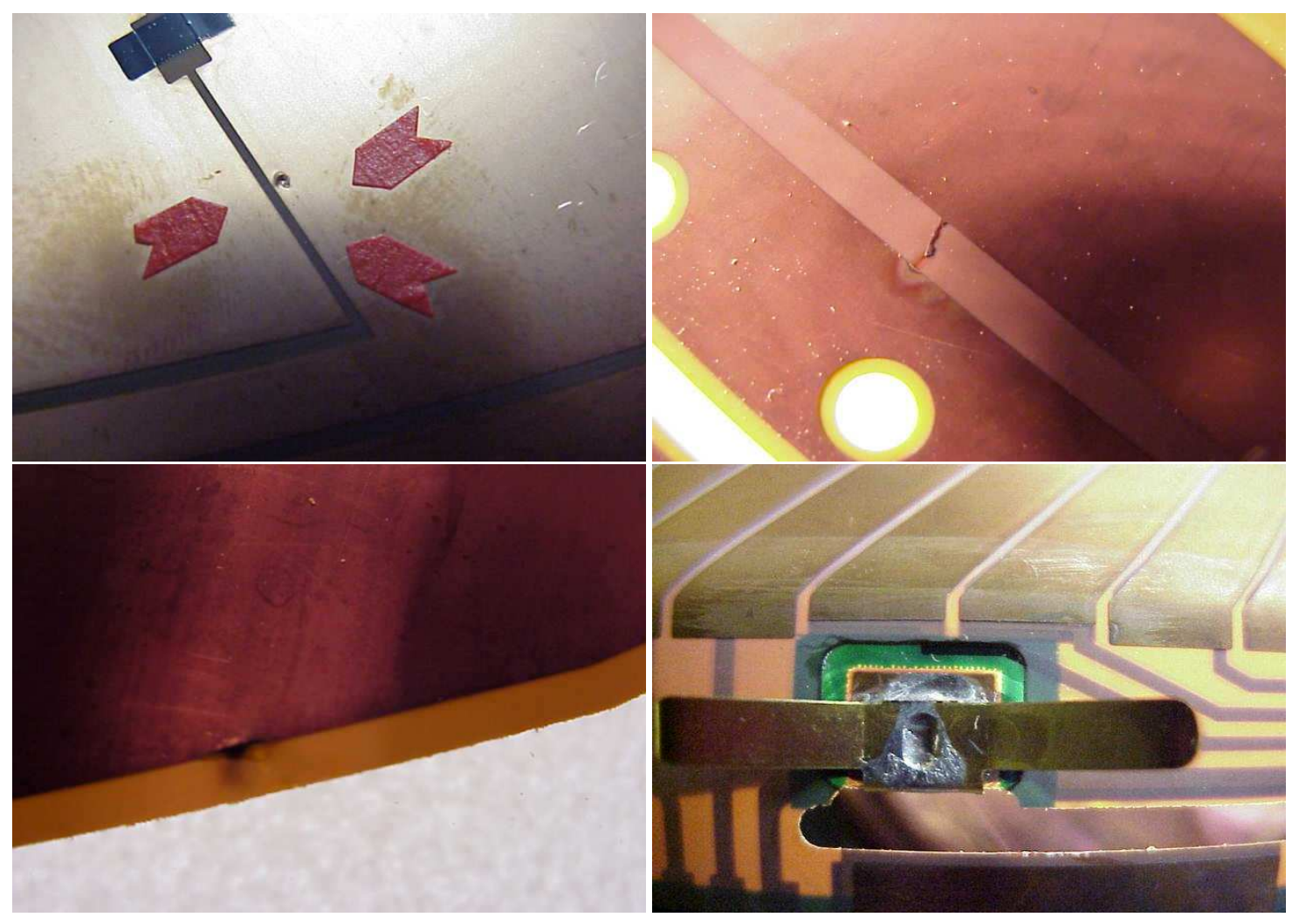

Figure 21: Pictures showing various high-voltage problems observed on pre-series electrodes: hole in polyimide insulation (top left), bad copper etching on HV layer (top right), bad gluing on electrode edge (bottom left) and signal layer trace not covered by isolating polyimide (bottom right). In this last picture one can see that the two central strips have been shortened to increase the distance from the inner layer. 


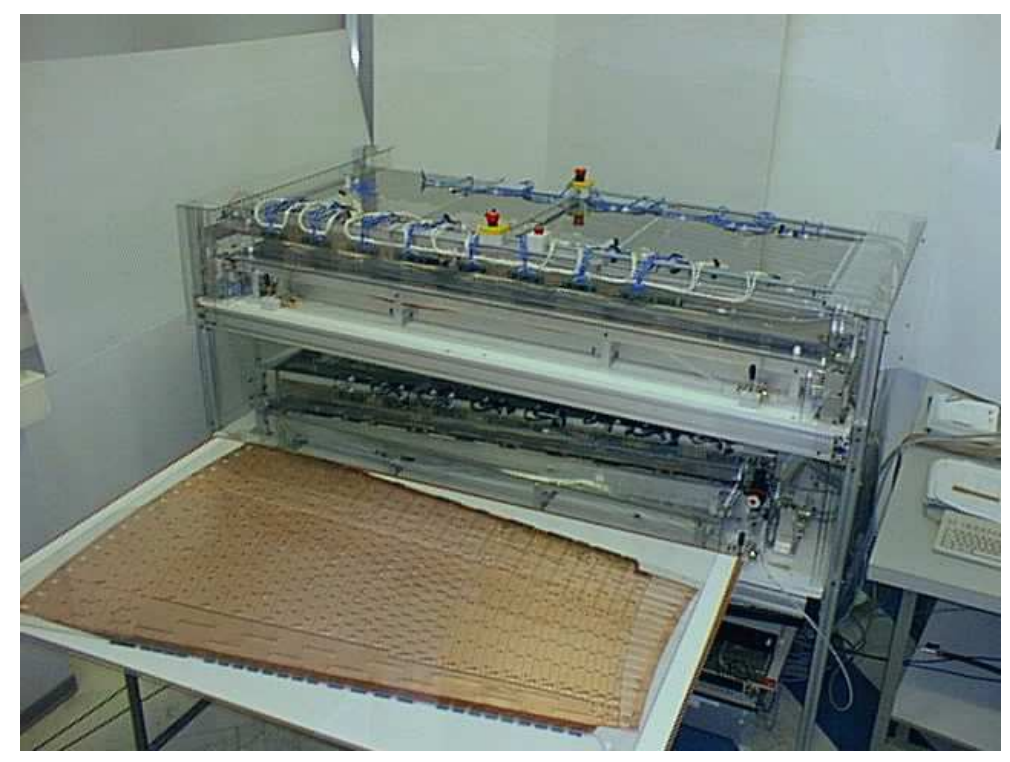

Figure 22: View of the flat test benches. The top part is used to test A and B electrodes, the bottom part is used for $\mathrm{C}$ and $\mathrm{D}$ electrodes. The electrode just about to be tested is a D electrode.

The principle of the measurements is the same for bent and for flat electrodes, the main difference being the different mechanical geometry of the bench used to perform the tests.

During series production, all electrodes were tested to ensure all electrical acceptance criteria (resistor values, electrical contacts, short circuits) were met for delivery.

Additionally this gives us the possibility to compare measurement results before and after bending, to ensure quality control before stacking of the electrodes. The main motivation for this comparison is the fact that potentially dangerous and evolutive cracks may appear on the resistive pads during handling or bending, so that an electrode conform at flat could be significantly degraded when it would finally be stacked. Such cracks can be detected because they tend to increase the value of the resistances.

Fig. 22 shows one of the flat electrode benches installed at the circuit manufacturer. This equipment was operated by the manufacturer's staff with substantial help for training and maintenance from the ATLAS collaboration institutes [10].

Principle of the measurements Resistances are measured with an automated multimeter (Keithley 2000), and capacitances with a RLC bridge. A combined measurement using only the RLC bridge was not feasible, since in the frequency range accessible with the RLC bridge, the resistive and capacitive component of the impedance are of the same order of magnitude.

The measurements are done with probes touching the copper pads on the electrodes, and also through the high voltage and signal connectors. The probe extremities have a flat or convex shape, to better follow the electrode shape for bent electrodes and to provide a good electrical contact between the electrode and the measurement system. Between the measurement apparatus and the probes, there is a multiplexing system that allows to connect to the multimeter and RLC bridge the needed combinations of probes. A sketch of the whole system is shown in fig. 23 (left), a schematic view of the mechanical structure of a bent electrode bench is shown in fig. 23 (right). The total number of measurements is of the order of 1500 measurements per electrode, and the duration of the test is about 20 minutes.

As an example of the use of the measurements to investigate the properties and to find the defects of the electrodes, we show in fig. 24 the capacitance measurements for one electrode of the D type. One can notice that the mean values are different on each side of the electrodes. The ratio of the mean values agrees with the ratio of the dielectric thicknesses on each side.

The capacitance values as a function of cell number, shown on fig. 25, can easily be understood in terms of cell surface variations as a function of $\eta$. On this kind of plot, eventually broken connections were detected by the fact that they give a capacitance value of zero.

Fig. 26 shows an example of the resistance measurements for one electrode as a function of cell number, in S1. Here again, the pattern observed can be traced back to the aspect ratio variation of 


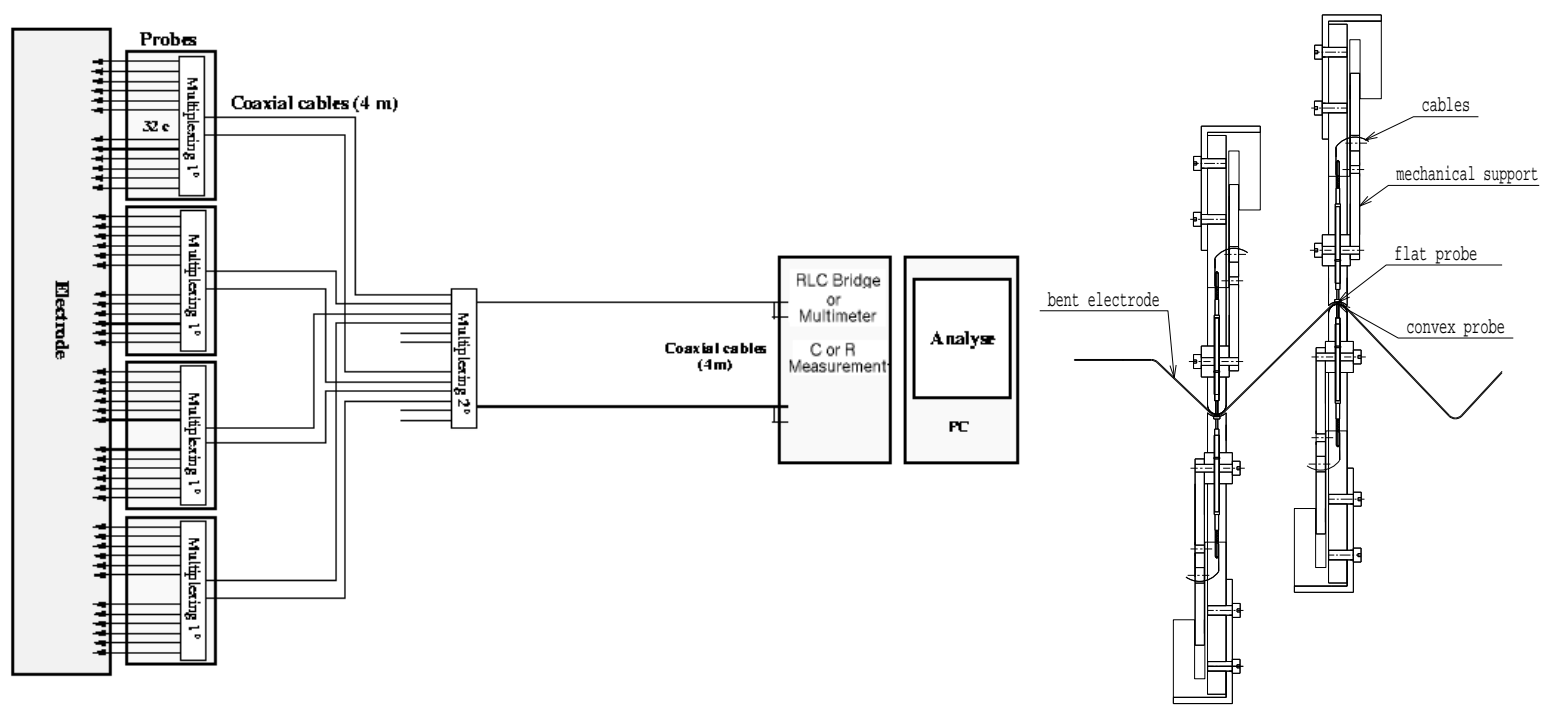

Figure 23: Left : schematic view of the data acquisition and multiplexing scheme used in the flat and bent benches. Right: detail of two test probes used on the RC bent electrode benches. The probes used on the flat electrode benches are very similar, only the position changes and also the shape of the tip of the probes.

the resistors as a function of $\eta$. In this case, the pattern observed at flat was compared resistor by resistor with the pattern observed on the bent electrode to spot increases in resistivity, signaling cracked resistors.

One of the major concerns in the production of these large circuits was the feasibility to serigraphy resistive ink over such a large surface in a well controlled, uniform and reproducible way. The mean value of the resistance obtained for each electrode is discussed in section 3.3.2. Fig. 27 shows the resistance values, normalized for the form factor (the resistivity), as a function of their location on the surface of the electrode. The pattern of fig. 5 for electrode A as seen from the back of the electrode can be recognized. The resistivity obtained is a property of the ink used but strongly depends on the details of the application method. Fig. 27 contains data for $\sim 300$ electrodes.

\subsection{Global production results, yield, evolution}

\subsubsection{Electrode production statistics}

As already mentioned, for equipping all the calorimeter modules, 6144 electrodes were needed. In Fig. 28 we show the delivery of all electrode types, over a period of two and a half years.

Of course more circuits were started by the manufacturer, but never made it to delivery. Once startup of production was over, a stable yield of $85-90 \%$ was obtained. Also a typical weekly delivery included 80-100 electrodes.

\subsubsection{Evolution of the resistivity with time}

Fig. 29 shows the average resistivity of the conductive ink over the whole production period (for one type of electrode). After some erratic variations at the beginning of the production, due to tuning of the process, the resistivity has been quite stable over two years of production. However, no satisfactory explanation has ever been found to the remaining variations, due to the multiple parameters involved in the final value of the resistivity.

\subsubsection{Resistor measurements at cryogenic temperatures}

Several measurements were performed in order to estimate the values of the serigraphied resistors on the electrode surface when immersed in liquid argon. Some trials to measure individual resistors in liq- 

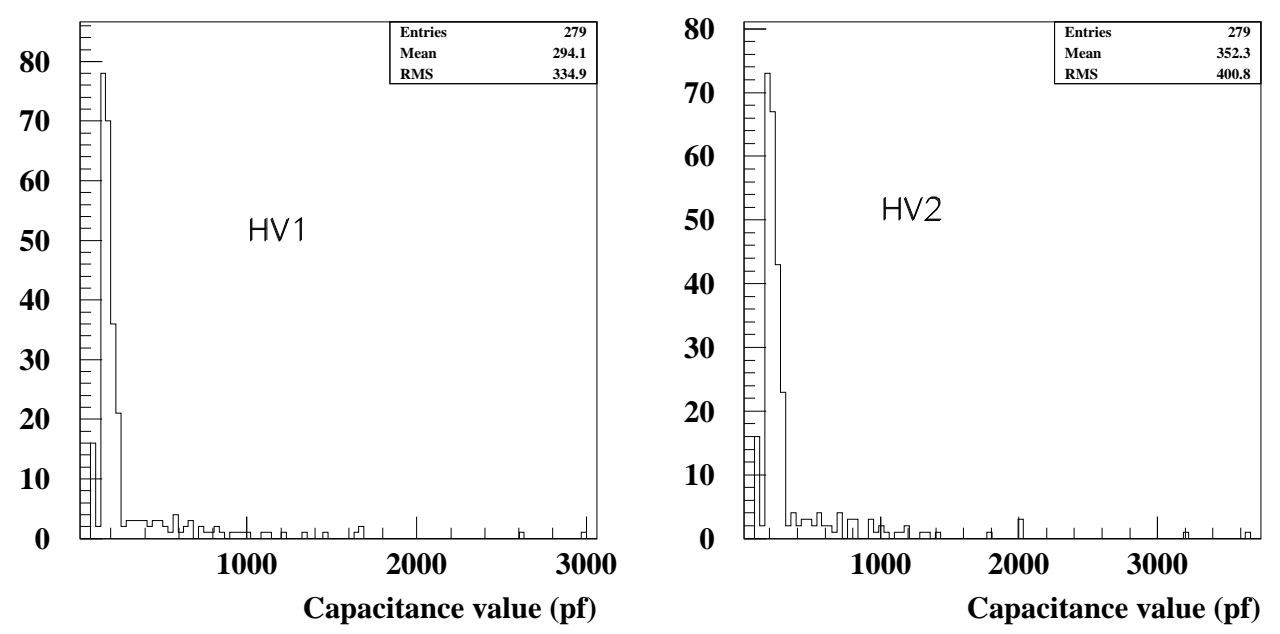

Figure 24: Distribution of cell capacitance measurement of one flat D electrode, on HV1 side (left), and on HV2 side (right).

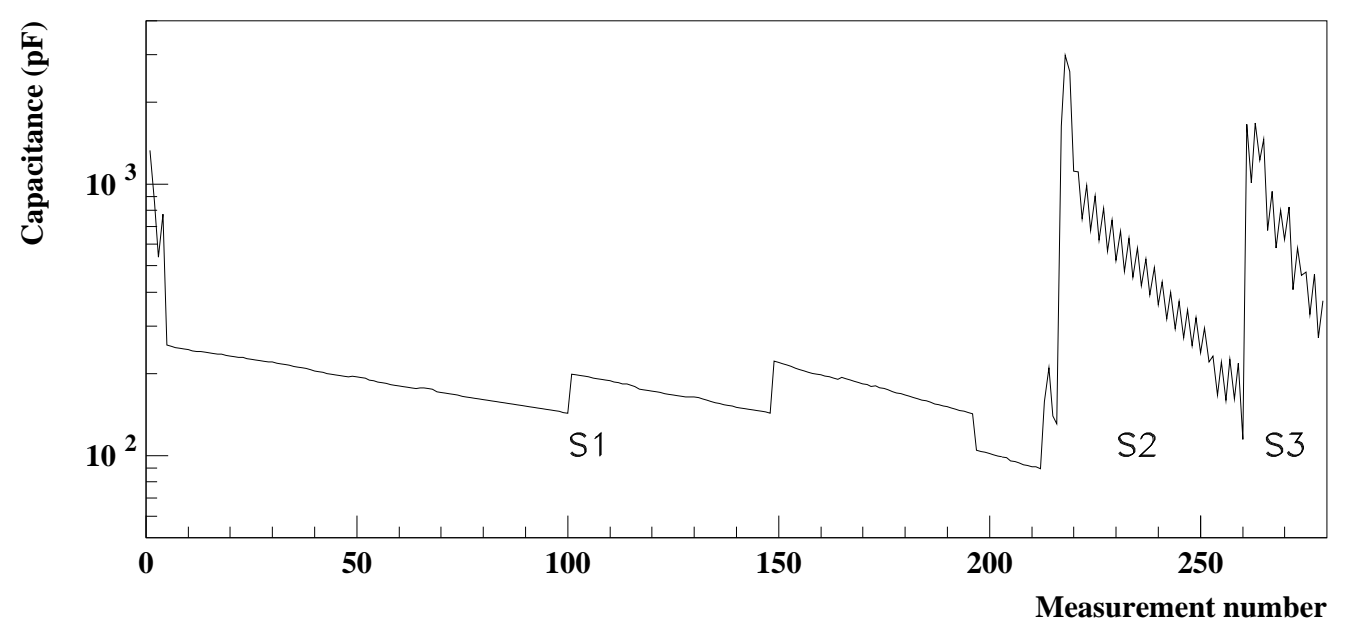

Figure 25: Capacitance measurements for a D electrode as a function of measurement number. In the three samplings, the measurements are ordered by increasing $\eta$ position. 


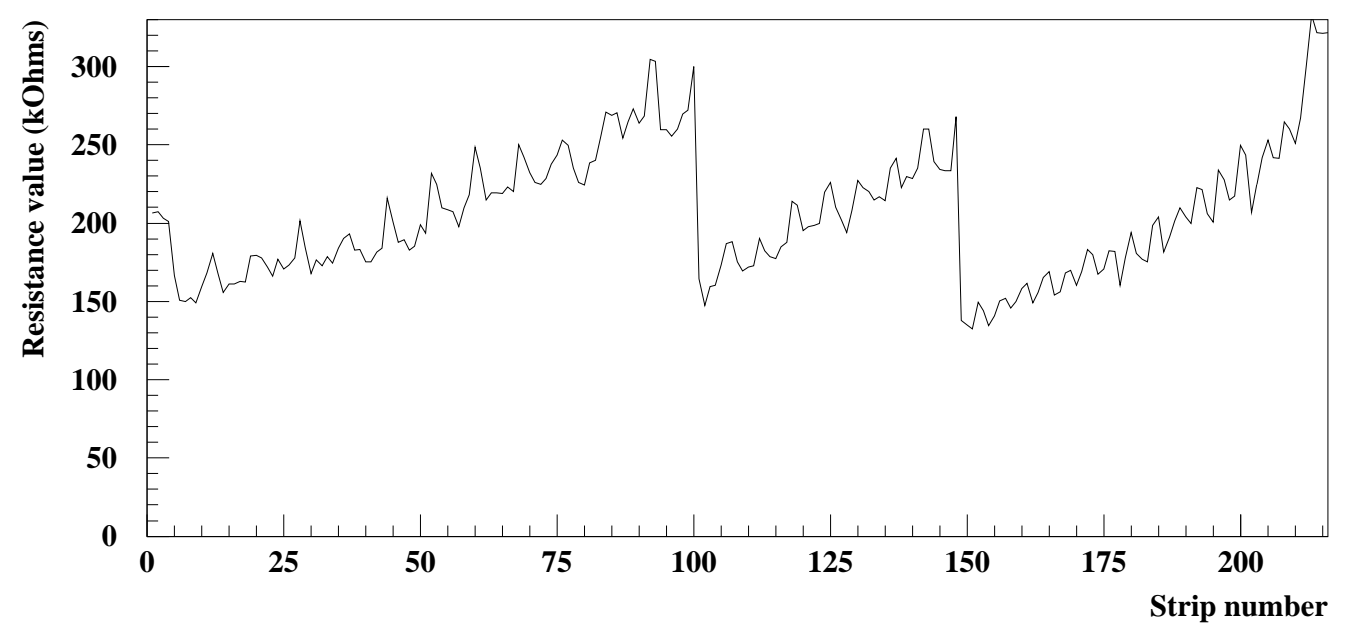

Figure 26: Resistance as function of strip number (sampling 1), for a D type electrode. Strip numbers are ordered in increasing $\eta$.

uid nitrogen with test points were found to give results with widely varying warm (room temperature, $300 \mathrm{~K}$ ) to cold (liquid argon, 90K) factors.

A more "system type" measurement was done measuring the resistance as would be seen from the HV source. Since it is not feasible to generate a current in the liquid argon gaps (as would be the case when particle beams are used), the resistors of a whole HV cell were ganged together and their resistance measured at several voltages or DC currents. Instead of placing 32 electrodes in parallel to the same source, groups of eight were formed such as to have four different measurements of ratios V/I. Also separate measurements were made for resistors on both HV faces (in the same way they have separate HV sources). The V/I ratios were monitored during cool down (and also when warming up). The evolution of the warm to cold ratio for one measurement can be seen in fig. 30 left. One measurement includes the parallel and serial connections of around 80 individual resistors. The equivalent resistor value in this setup goes from some $10 \mathrm{k} \Omega$ to $50 \mathrm{k} \Omega$ and the ratios $\mathrm{R}(\mathrm{T}=90 \mathrm{~K}) / \mathrm{R}(\mathrm{T}=300 \mathrm{~K})$ obtained are $5.06 \pm 1.6$ for one HV face and $5.16 \pm 0.6$ for the other face.

It is also interesting to note that the warm to cold ratio obtained changes by a small amount when the current is measured at different voltages. This is shown in fig. 30 (right) where voltage points are shown over two orders of magnitude. The application within ATLAS functioning at nominal luminosity would produce currents equivalent to a voltage drop around one volt in this plot.

\subsubsection{Resistor evolution under irradiation}

A handful of individual silk screened resistors were irradiated to $10^{13} \mathrm{n} / \mathrm{cm}^{2}$ which is equivalent to ten years of ATLAS operation in LHC. Some of them had been deliberately damaged and repaired (see below, section 4.2.4) in order to test the behavior under radiation of the repair method. Resistance values were measured before and after irradiation. For standard resistors a small increase in resistance $(\sim 3 \%)$ was observed. Repaired resistors increased in mean by $\sim 10 \%$, indicating the appearance of a non-zero resistance due to irradiation of the silver based epoxy lacquer used to fix the damages. This increase is acceptable given the rather wide range allowed for the resistivity (see section 2.1.4). 

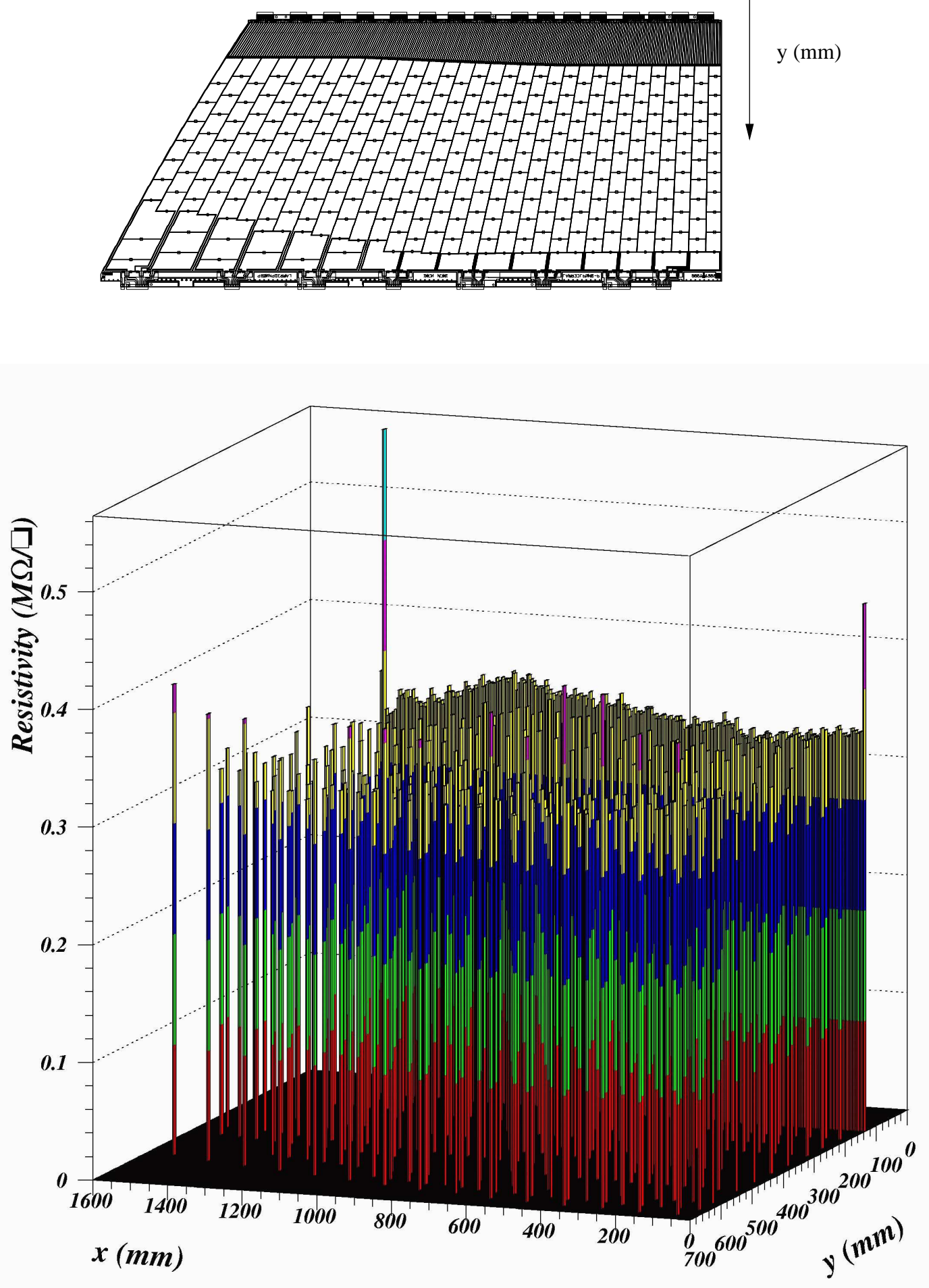

Figure 27: Resistivity of the pads distributed over their location on the electrode surface. Data is the mean value over $\sim 300$ A electrodes. 


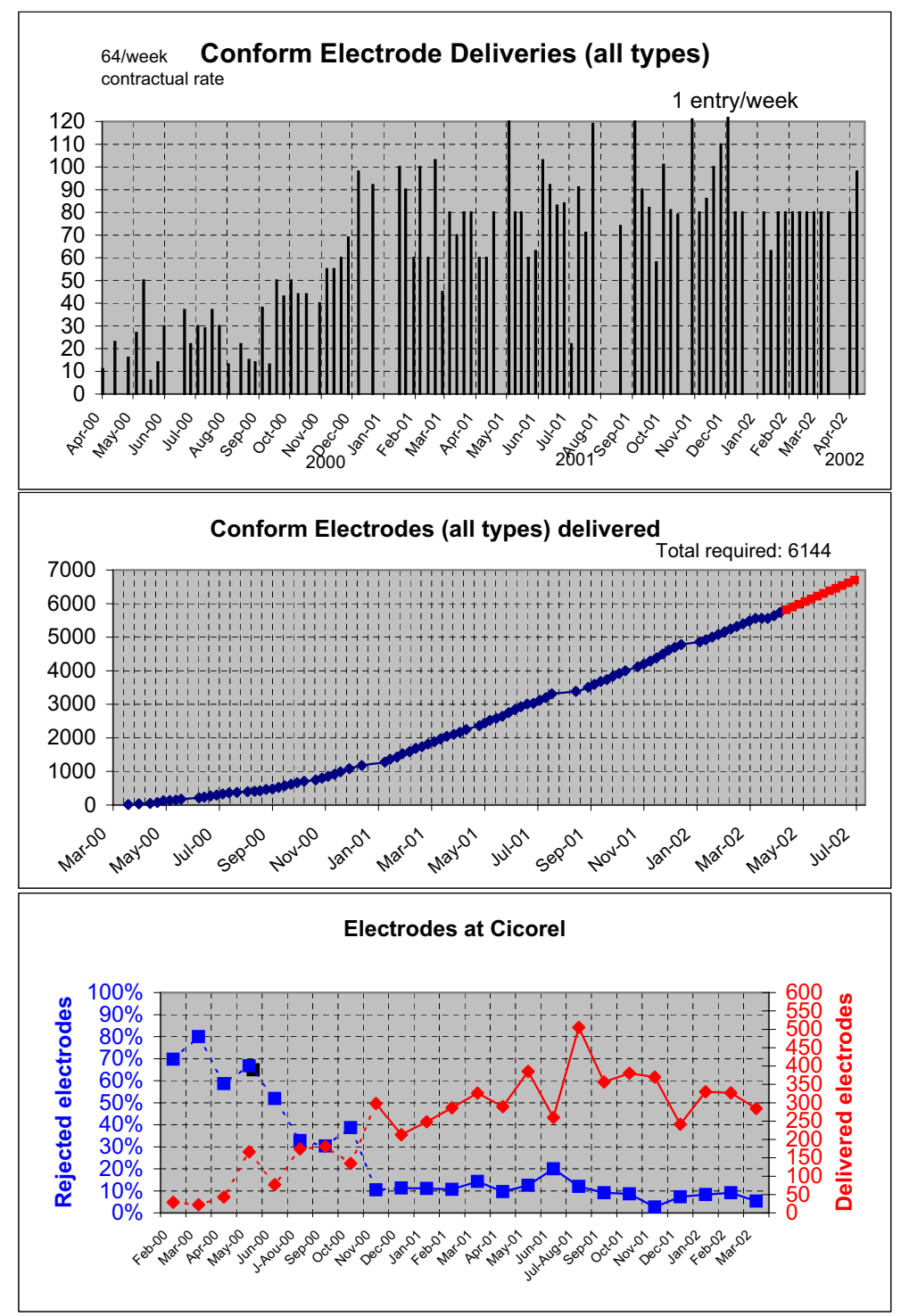

Figure 28: Delivery of electrodes over time (all types). Note the weekly delivery rate (top) and yield at manufacturer (bottom). 

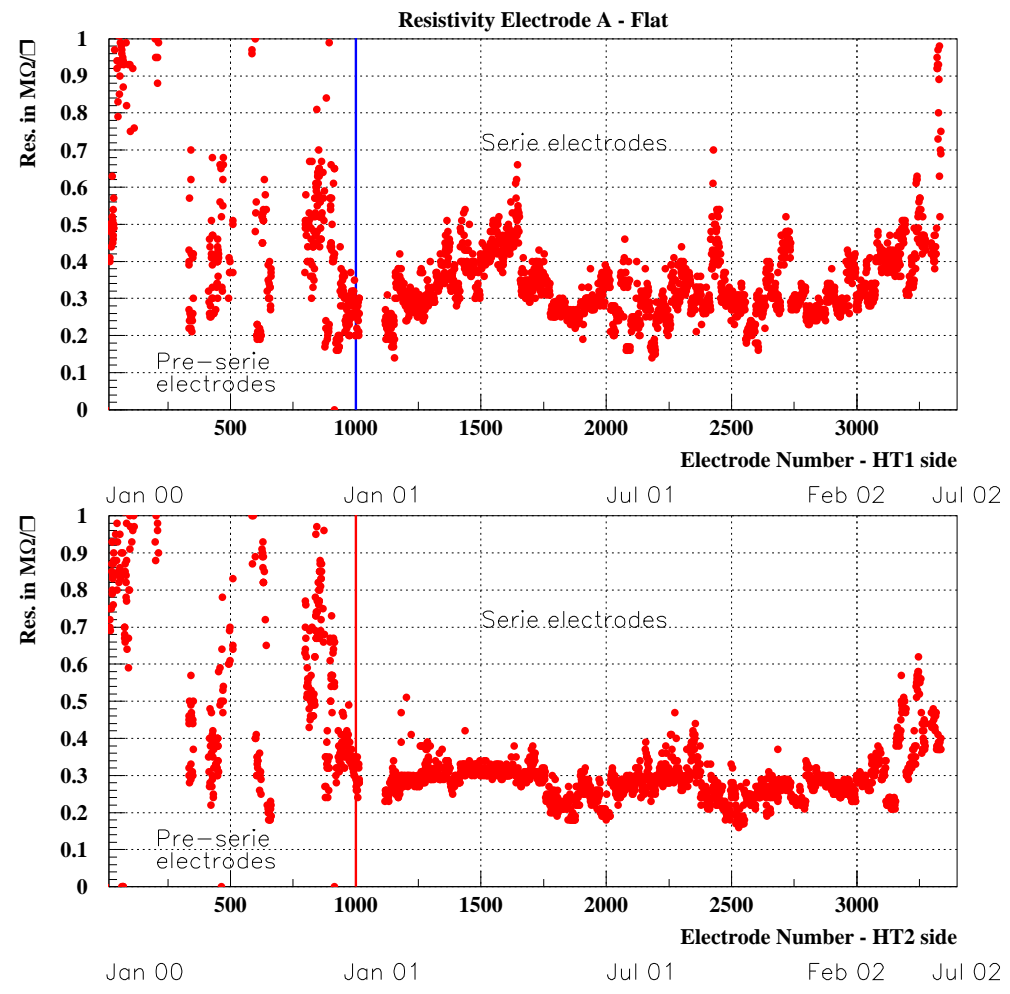

Figure 29: Resistivity of the electrodes as function of production date
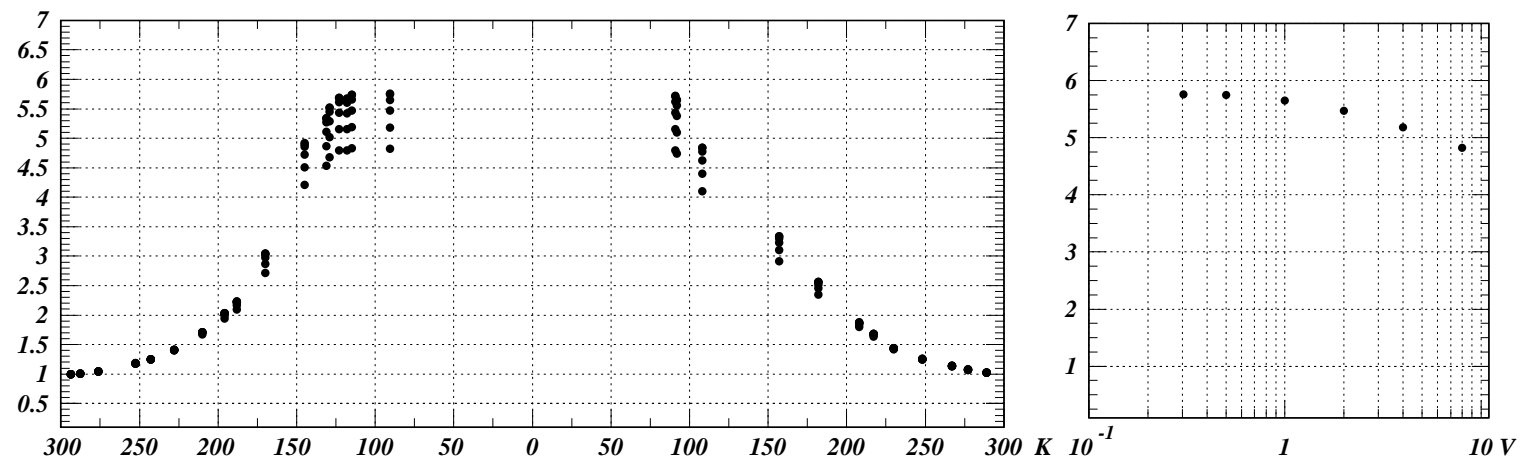

Figure 30: Left: evolution of the resistance between ambient and LAr temperatures. The measurements were performed while cooling down and warming up again and are normalized at room temperature. Right: Ratio $R(T=90 K) / R(T=300 K)$ and its dependence on the voltage used to measure it. 


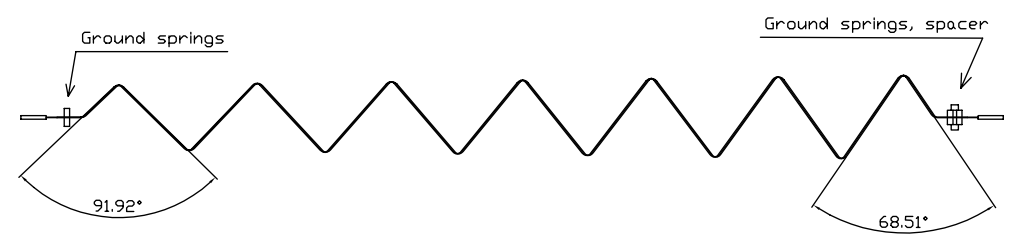

Figure 31: Profile of a barrel electrode showing the changing folding angles

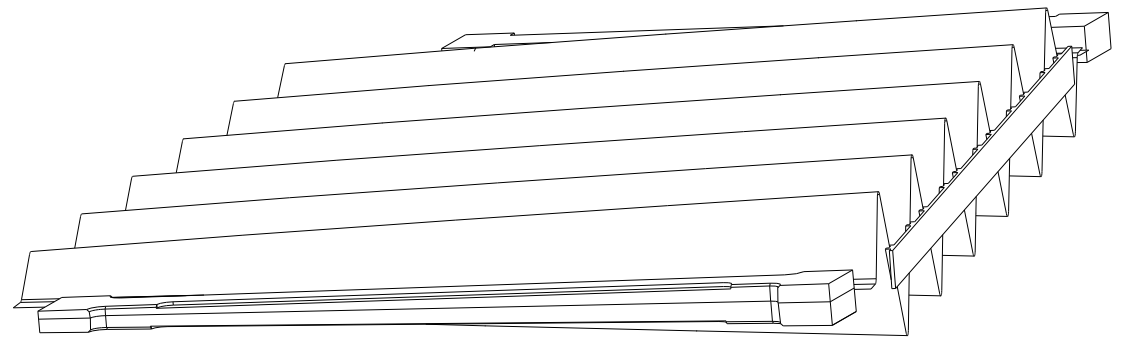

Figure 32: An end-cap inner wheel absorber. The changing angle folds can be appreciated

\section{Electrode Bending and finalization}

\subsection{Accordion bending}

Electrodes as described above were produced and delivered from industry as flat circuits. An important and delicate step is the forming of the electrodes to the final accordion shape. Due to the quite different geometry of the barrel and end-cap detectors, two different approaches were followed since the beginning.

- The barrel geometry follows bends all parallel to the electrode edges. Each bend has the same bending radius but a slightly different angle and increasing wave amplitude, thus making a geometry which opens up in the calorimeter radial direction. An electrode profile can be seen in fig. 31 .

Thus it is possible to start bending from one electrode edge and generate one fold after the other. A machine was designed and built with pneumatic actuators moving vertically individual knives onto a bottom "mold" which was machined to the final electrode shape, i.e. close to the neutral fiber of the accordion. The electrode is precisely positioned in between the machine jaws by means of alignment holes made at the same time as the cutout. Actuators are operated sequentially making folds at increasing radii. Since the shape of the press is made to be the theoretical shape of the accordion, the elasticity of the flexible electrode laminate tends to increase the fold angles. To compensate for this effect, electrodes are cured $\left(\sim 160^{\circ} \mathrm{C}\right)$ for a few hours in elastomer molds thus fixing the shape of the electrodes to the right geometry.

- In the end-caps the electrode folds are all equal but their amplitudes and angles vary along the fold. A press to fold this geometry was built for accordion shaping the absorbers, with moving upper and lower knives. This solution was considered too complicated for deforming the copperpolyimide material making the electrodes. The accordion geometry of an inner wheel absorber is shown in fig. 32.

Another approach was chosen [11] profiting from the fact that all folds are equal. A single knife, is pressed against a (rather hard) horizontal rubber base by a conventional folding press. The knife is fixed with a tilted angle to the press jaws thus deforming the material at a changing angle. The position of the fold is determined by holes made on electrode excess material defining the summit line of the bend. The holes are complemented by a cutout shape in such a way that after all folds have been made, the excess material can be removed by a hand cutter and the electrode has its final shape and size. 
With this method, the folds can be "over-bent" in such a way that after removing the knife constraints, the required final shape is obtained without any need for material curing. Of course quite some parameters had to be found out experimentally like the knife depth vs. fold angle obtained, the relaxation of the material and the special tuning for the first and last half-folds, but once the laminate of the electrodes and its autoclave gluing was in series production, this folding method was seen to give precise and well reproducible results. The bending of the end-cap electrodes was performed in industry ${ }^{11}$.

In fact this method was so satisfactory that the original barrel electrode bending press was abandoned and a method similar to the end-cap single-knife approach was used to bend over $2 / 3$ of the barrel electrodes. The main advantages thus obtained were a more precise final shape and a faster bending rate since no curing cycle was required anymore.

The electrode span between the summit of the outermost folds, measured at several $\eta$ (or $\mathrm{z}$ (r) for the barrel (end-cap) electrodes) positions is a good global parameter which describes the quality of the bending. It was measured for each electrode and traced in the production database. Fig. 33 shows a distribution of the span of more than 1500 A type electrodes bent with the single-knife method over a two year period. Two measurements are performed at two z positions $z \sim 0$ and $z \sim 1500$. While the nominal value is $435 \mathrm{~mm}$ (shown as a line on the histograms), the bending tuning targeted a span a few $\mathrm{mm}$ shorter such that for stacking the electrodes on the absorbers, a small pull across the folds fits (and clamps) the electrodes on the absorber G10 bars.

\subsection{Final commissioning}

\subsubsection{Equipment}

The electrodes are equipped with ground contacts and spacers on the back side (A and B type only). This installation is one of the last operations before the electrodes are sent to the assembly sites for module stacking since the bending of the electrodes requires the electrode surface to be perfectly flat.

The equipment is performed at the testing sites together with a series of other routine operations. The sequence of the operations is the following:

1. electrode reception and inspection;

2. RC test;

3. ground contacts equipment;

4. resistor repair;

5. RC re-test;

6. spacer mounting (only for A and B electrodes);

7. cleaning;

8. HV test;

9. cut out of the additional pieces used for mechanical reinforcement of the structure during handling and testing (barrel electrodes only);

10. final cleaning and packing.

The sequence of operations 2 and 3 can be inverted. The ground contacts are soldered to the copper pads on both sides of the electrodes with a Sn-Pb (60-40) alloy with a low eutectic point $\left(180^{\circ} \mathrm{C}\right)$ using a large solder tip at $230^{\circ} \mathrm{C}$ powered by a $60 \mathrm{~W}$ supply. This procedure proved to preserve the integrity of the electrode.

All manipulations of the electrodes are made with gloves and using rigid supports to avoid stresses to the electrodes and in particular to the resistors pads.

The test and equipping areas are maintained at a good level of cleanliness but they are not strictly controlled areas. Particular care was made in the packaging of the outgoing electrode batches to prevent damages due to a mishandling during transport.

A paper document where all the operations performed are logged is attached to each electrode. The electrode logistics and the results of the tests are maintained in a central database.

${ }^{11}$ RIPM, Saint Cannat, France 

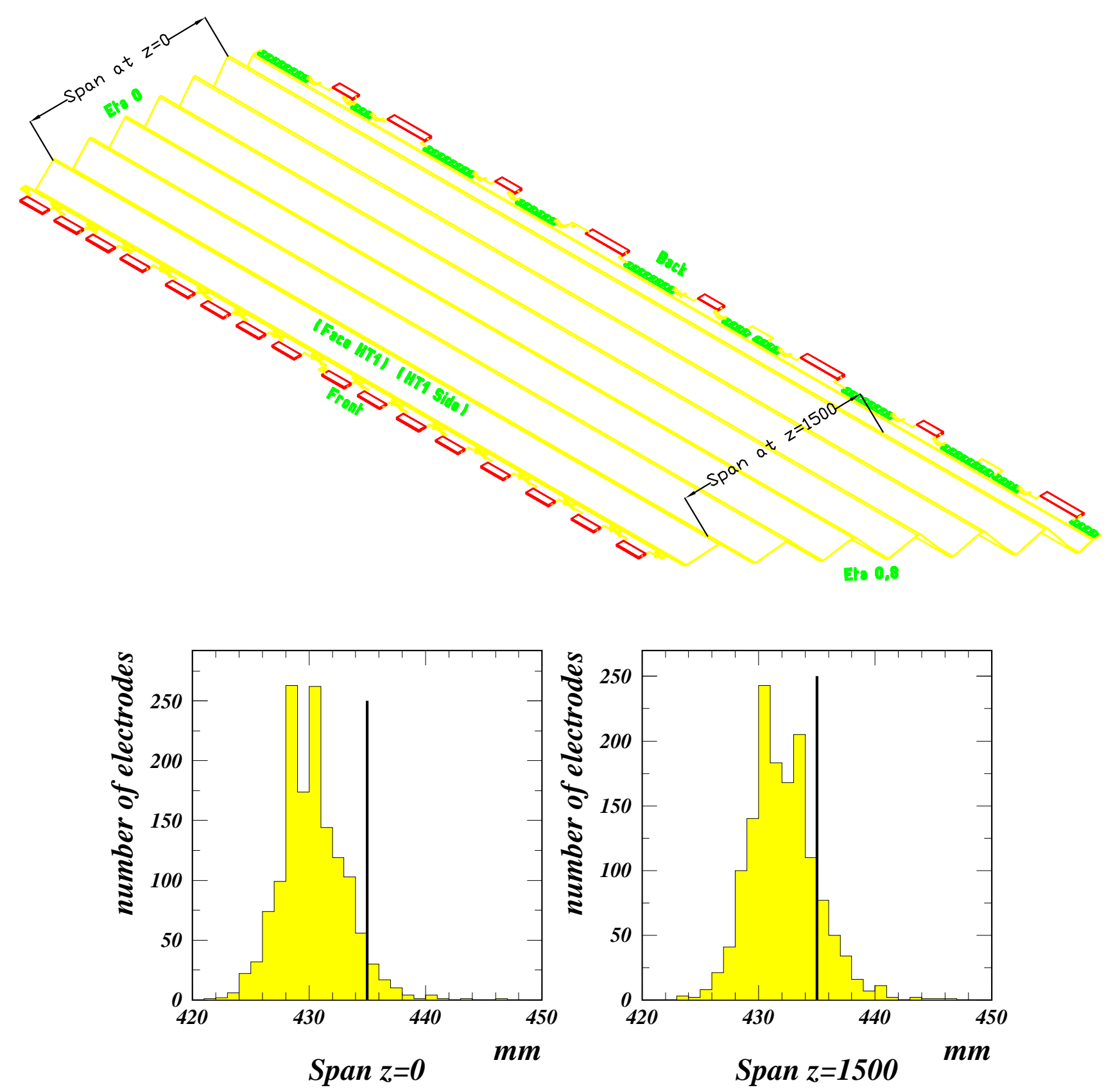

Figure 33: Span of folded A electrodes measured for more than 1500 A type electrodes at two different $\mathrm{z}$ positions. Note the obtained span width which was intentionally lower than the nominal (vertical lines) in order to ease the electrode stacking. 


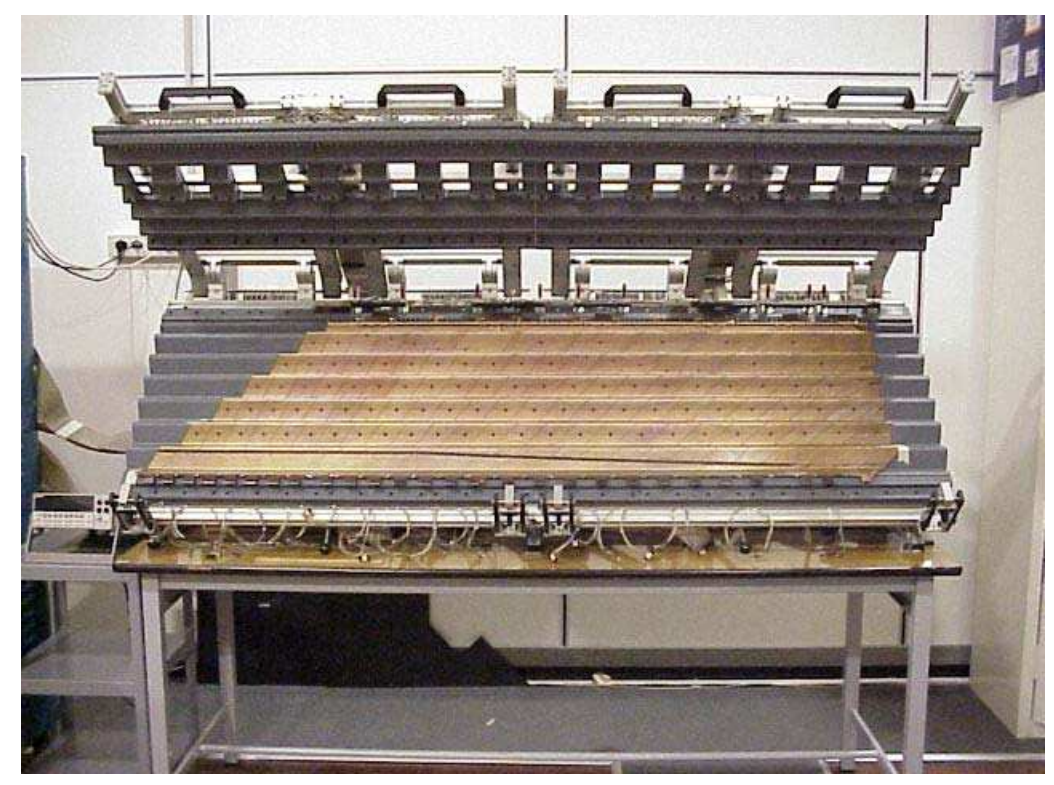

Figure 34: Bench for performing final RC tests on B electrodes

\subsubsection{HV Test Procedures at the Equipping Sites}

At the end of the equipping process, before they are shipped to the stacking sites, the electrodes have to undergo a new high-voltage test.

The test is similar to what is done at the production company site but it is more stringent. It articulates in three different tests.

The first test is similar to the one described in section 3.2.3 with the two following differences: the test lasts 12 hours (instead of 1 hour) and the electrode is brought to $2500 \mathrm{~V}$ (instead of $2000 \mathrm{~V}$ ).

In the second test the high voltage $(2500 \mathrm{~V})$ is applied to one of the HV layer only while both the signal layer and the other HV layer are kept to ground. This test is performed in order to exclude the presence of arcing conductive paths between the two HV layers.

In the third test, the odd (even) HV sectors of the HV1 (HV2) side of the electrode are kept to $1000 \mathrm{~V}$ while the other ones are kept to ground.

The reason for these last two tests, which might appear useless as the design foresees to operate both $\mathrm{HV}$ layers at the same high voltage, is the following. In the ATLAS detector, for what the high voltage is concerned, each calorimeter half barrel module is divided into 7 high voltage sectors of dimension $\Delta \eta \times \Delta \phi=0.2 \times 0.2: 4$ sectors in the A electrodes and 3 sectors in the B ones. Each sector consists of 32 electrodes. A similar partition holds for the end-cap modules. All HV1 sides are chained together and biased by a single high-voltage power supply. The HV2 sides are connected to a separate high-voltage source. This is a compromise between the need to limit the number of power supplies and the need for some safety margin. Indeed, if some problem (e.g. short) develops on one side only of one electrode, forcing the high voltage to be turned down or kept at a lower than nominal value, it is still possible to operate the affected sector by having the high voltage on all the other semi-gaps and then correcting offline for the signal not collected in the other half of the sector. The last two tests ensure that this kind of emergency operation is possible without discharges between contiguous sectors, semi-gaps or between electrodes on contiguous modules kept at different voltages. The $1000 \mathrm{~V}$ operating voltage has been chosen because the distance between cells in neighboring sectors is about $0.5 \mathrm{~mm}$ in the strips and $1 \mathrm{~mm}$ in the middle. At these distances, a tension of $2000 \mathrm{~V}$ would have caused discharges.

\subsubsection{RC Finalization}

The benches used for the final commissioning of the electrodes are very similar to the flat electrode benches except for the mechanical supports in accordion shape. For example, fig. 34 shows a photograph of the bench used to test bent $B$ electrodes. The electronics and data acquisition systems are exactly the same, to ensure that one bench could serve as a source of spare parts for others in case of emergency. 
Average number of repairs per electrode per batch

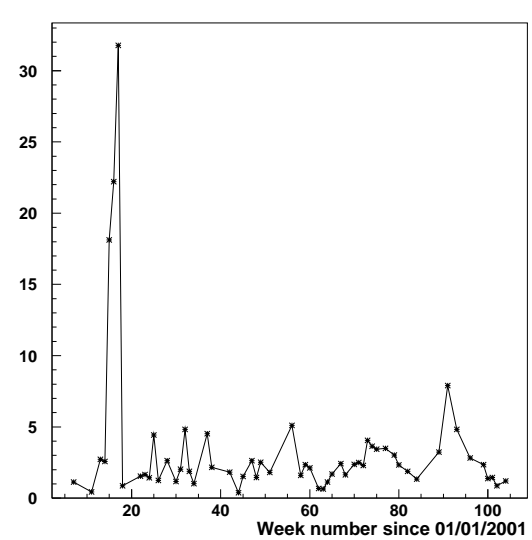

Number of repairs per electrode

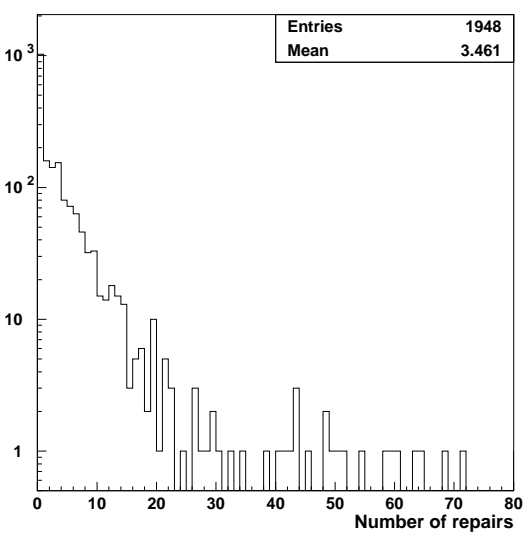

Proportion of electrodes with no repair

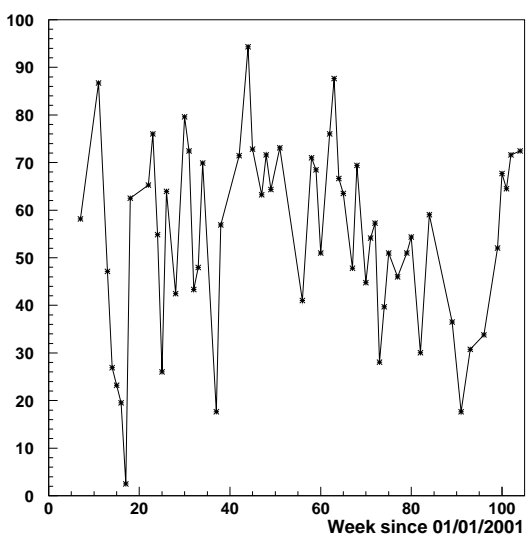

Figure 35: Resistance repair statistic per weekly batches. Left and middle: Average number of repairs per electrode. Right: percentage of electrodes needing no repair over the whole production and test period. An A electrode typically contains 1000 resistors. An electrode needing more than 20 repairs was discarded.

\subsubsection{Repairs of silk screened resistors, statistics}

Since it was not possible to ensure that after all the handling and bending operations starting from the flat electrodes none of the resistors gets cracked, a systematic check was performed, by remeasuring all the resistor values after equipment of the electrode, and by comparing the results with the measurements obtained at flat. If the ratio between the bent and flat measurement values was above a given threshold, the resistor was considered as cracked and had to be repaired. The threshold was first set at 3 , since an increase of a factor of 3 leads to a crack that could usually be seen under binoculars. However, an increase of less than 3 already indicates a weakened resistor, that would be wise to repair. With these considerations in mind, we chose to decrease the threshold to a factor of 2 as soon as the quality of the electrode production and bending had sufficiently improved. If the total number of damaged resistors was above 20, the electrode was considered to be too badly damaged and was put aside.

To repair a resistor, it was first checked visually under binoculars, and the crack was painted over with silver loaded epoxy conductive paint ${ }^{12}$. The lacquer had to be cured for 15 minutes under $130^{\circ} \mathrm{C}$ air flow (or 45 seconds at $175^{\circ} \mathrm{C}$ ) . After these operations, the electrode was retested and if necessary, the procedure was repeated until no more repair was required. Cryogenic and radiation tests of the repaired resistors showed a good reliability of this procedure.

Fig. 35 details repair statistics for electrodes of type A: the mean number of repairs per electrode as a function of time, the distribution of the number of repairs as well as the fraction of electrodes needing no repair. The data is shown grouped per batch of one week of workload at the testing and equipment facility. As for the resistivity variation with time (section 3.3.2), it proved very difficult to trace particularly good or particularly bad batches to a specific reason.

Seldom, shorts between copper pads of the HV electrode layers were observed, both on flat and bent electrodes. These shorts were easily removed by local application of a solution of $\mathrm{FeCl}_{3}$ to etch the unwanted copper traces.

\section{$5 \quad$ Electrode Stacking}

Electrodes once produced, bent, equipped and tested are delivered to stacking sites. In a certain sense once stacked in modules, the electrodes arrive at their final destination. They are stacked onto calorimeter modules, the unit of calorimeter construction for practical handling, cabling and testing. The modules are then mounted into "wheels" to be inserted in the cryostats. All module assembly aspects are covered in detail in [12] for the barrel part. We discuss two points in which the electrode properties directly affect the quality of the stacking.

${ }^{12}$ Epotek H20E 


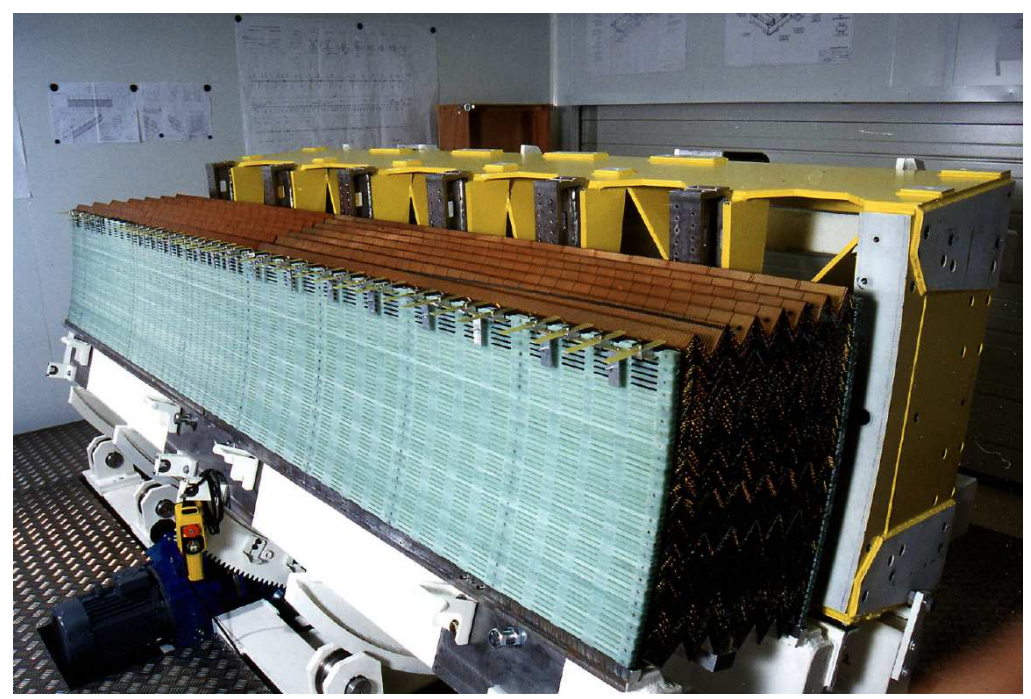

Figure 36: Stacking frame with barrel module in process of stacking.

Fig. 36 shows a barrel module on the stacking frame in the process of being stacked. We can see the $\mathrm{A}$ and $\mathrm{B}$ electrodes side by side.

\subsection{Module bulging}

The thickness of the liquid argon gap is precisely defined by means of the G10 bars (Al or composite rings) support structure for the barrel (end-cap) absorbers [1].

The electrodes and absorbers are interleaved with honeycomb spacers to ensure electrical isolation at high voltage. The thickness of the spacers has been chosen such that under normal conditions the electrodes should be able to move in the gap and not be clamped by the net-absorber stack. This is meant to allow a free differential shrinking of electrodes relative to the absorbers during cool-down. If this is correct, the stack of electrodes-absorbers piled horizontally, adopts the position given by the gravitational sagging of the absorbers. This is shown in fig. 37. The measured quantity is the overheight of the 4 th fold relative to the plane defined by the first and eighth folds. The measurements are performed at different $\mathrm{z}$ positions on the absorber. Since the bottom of the stack is held in the mounting jig, the first planes show no bulging/sagging. After some planes, enough tolerance space adds up so that the absorbers start to sag. After some more gaps, with even more free stacking height, the absorbers have reached their natural position and the sagging is constant.

Initially some electrodes showed some discrepancy from the nominal shape, in particular in the bending radii, summit positions and folding angles. Modules made with these electrodes showed a positive bulging. Several modules of this type were stacked at the beginning of the process. Still it was possible to mount them together to form a wheel since the whole stack is a rather flexible mechanical system.

\subsection{Capacitance measurements}

Once a module is stacked it undergoes several tests and measurements before the cabling phase starts. The measurement of the capacitance of the gaps, i.e. between the ground plane of the absorbers and the signal plane of the electrodes gives an independent measurement of the gap thicknesses. The gap thickness non-uniformity contributes to the constant term of the calorimeter energy resolution. Fig. 38 shows the measurements performed on a module of the barrel calorimeter. The capacitance is measured for eight sectors which correspond to the HV sectioning (except S8 which is normally fed from the HV of S7, but it is separated for the capacitance measurement). The absolute value of the capacitance is of course proportional to the sectors surface, but gap to gap variations are representative of possible stacking difference which could be caused e.g. by electrodes bulging the gap. The sum of the eight capacitances give a mean value per gap which we see is well contained in a $1 \%$ distribution. 


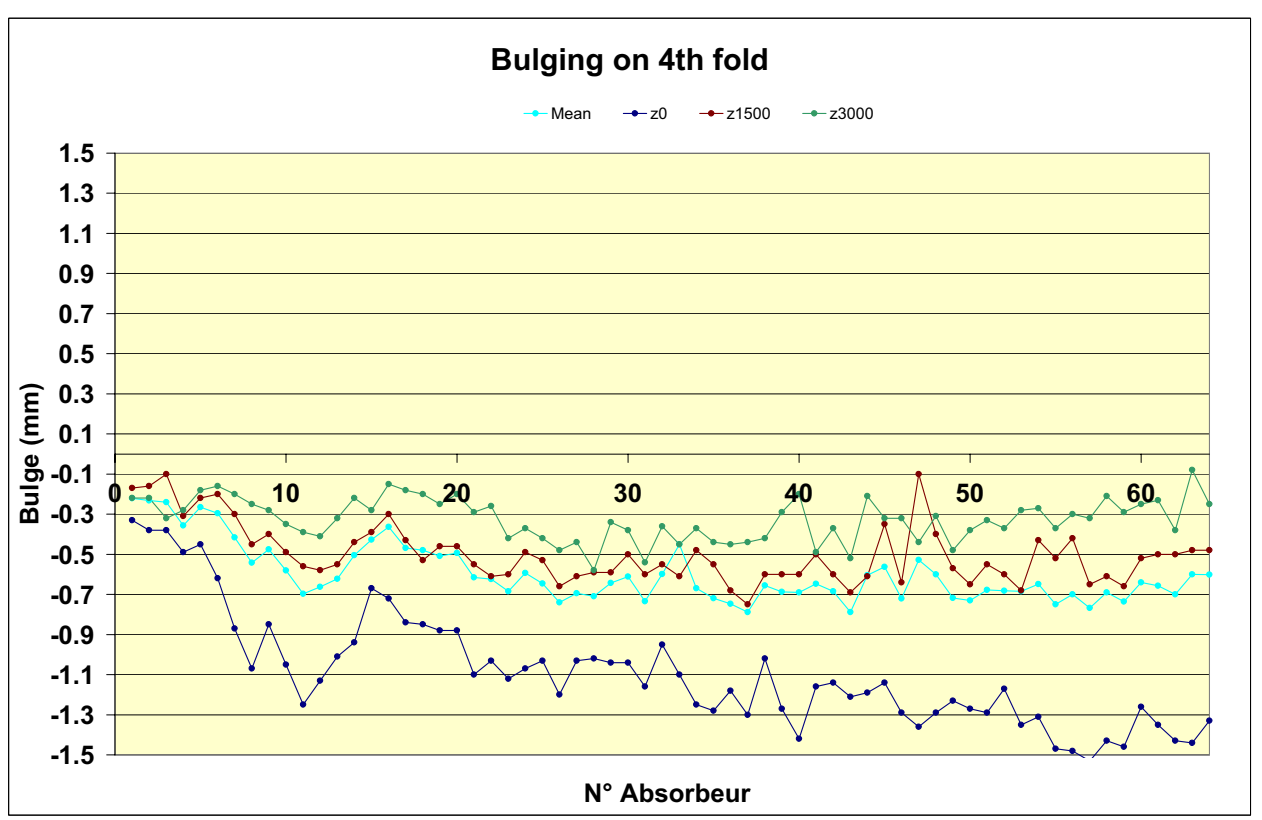

Figure 37: Over-height of 4th summit relative to outermost folds as a function of the number of the absorber or gap layer, for a barrel module.

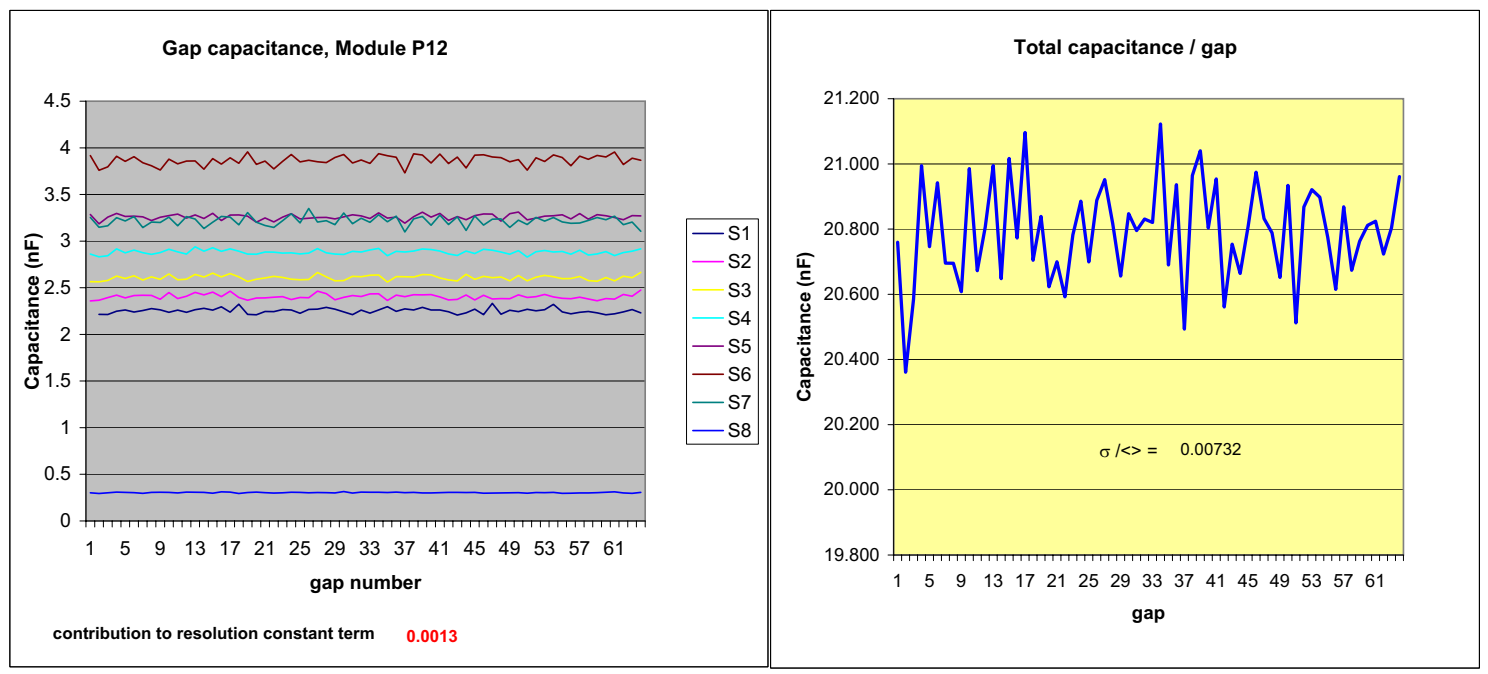

Figure 38: Gap capacitance measurement 


\section{Selected Full module results}

In this section we describe some measurements which have been performed in test beams or elsewhere. These measurements which concern the final performance of the calorimeter, constitute a global check of the quality of the electrodes.

\subsection{Resistive crosstalk}

The silk screened resistors which connect the middle and the front section of the electrode induce a resistive crosstalk [13]. The crosstalk has the same shape as the signal itself [8]. Fig. 39 (top) shows the crosstalk measured for all strips. We can see the value for several production modules and several sectors in $\phi$ being well below the $0.2 \%$ level. The lower part of fig. 39 shows a zoom over 40 strips where we see the strong correlation between the crosstalk and the value of the resistor measured directly on the electrode, at warm. The resistor value comes from values measured at the equipping and testing station and combined in parallel for the 16 electrodes forming up the cells. The pattern is due to the design of the electrode and the higher resistance values (lower crosstalk) follow well the silk screened resistor shape.

The ratio between these two traces would indicate a warm/cold factor of 1.2-1.3 for the resistors, in contrast to the larger factors mentioned in section 3.3.3. We note here that these lower ratio factors measured with high frequency signals are not the most relevant. In ATLAS under particle flux, current flows through the resistors in continuous "DC mode", in a similar way to the DC measurements of section 3.3.3.

\subsection{Test beam measurements}

\subsubsection{Position measurements resolution}

Thanks to the narrow strips of the first section of the electrode, the position resolution in the front is constant along $\eta$ and typically amounts to $0.1510^{-3} \eta$-units. In the middle section, the position resolution is $0.3510^{-3} \eta$-units. These two measurements [14], [9], are shown in fig. 40. They are in agreement with simulations and ATLAS specification. Another way to view the same calorimeter quality is to see the energy profiles (in units of strips) of $\pi^{0}$ s decaying into two photons separated by a given distance. In the present case, this was done using couples of test-beam photons to construct $\pi^{0}$ 's according to a kinematical simulation, at $p_{T}=50 \mathrm{GeV} / \mathrm{c}$ and $\eta=0.7$. Fig. 41 shows the energy profiles of the two photons generated at $0,3,6$ and $9 \mathrm{~mm}$ separation. At $6 \mathrm{~mm}$ separation, the energy sharing between the two most energetic strips starts to be swapped.

\subsection{LC uniformity}

Fig. 42 shows the inductance as deduced from signal shape studies for middle compartment cells. The pattern is directly related to the shape of the cells as drawn on the circuit including the connection strips as discussed in section 2.1.3. We show a comparison to an electrically simulated value from the circuit layout.

\subsection{Energy response and $\eta \phi$ uniformity}

As a final global result (see [15]), fig. 43 shows the energy response to $245 \mathrm{GeV}$ electrons of a barrel calorimeter module where the beam was directed systematically at each cell along $\eta$ and $\phi$. A cluster of $3 \times 3(\Delta \eta \times \Delta \phi=0.075 \times 0.075)$ middle cells around the most energetic one is summed for each point. Different rows of $\phi$ are shown in different colors. A total module non-uniformity below $0.6 \%$ is obtained. For local regions of $\Delta \eta \times \Delta \phi=0.2 \times 0.4$ it is less than $0.5 \%$.

\section{Conclusions}

The ATLAS Electromagnetic Calorimeter is equipped with large size electrodes made on flexible substrate. This is the concluding step of a long process of development, prototype production and industrial mass production where the large sized circuit design, the careful choice of materials as well as the many 

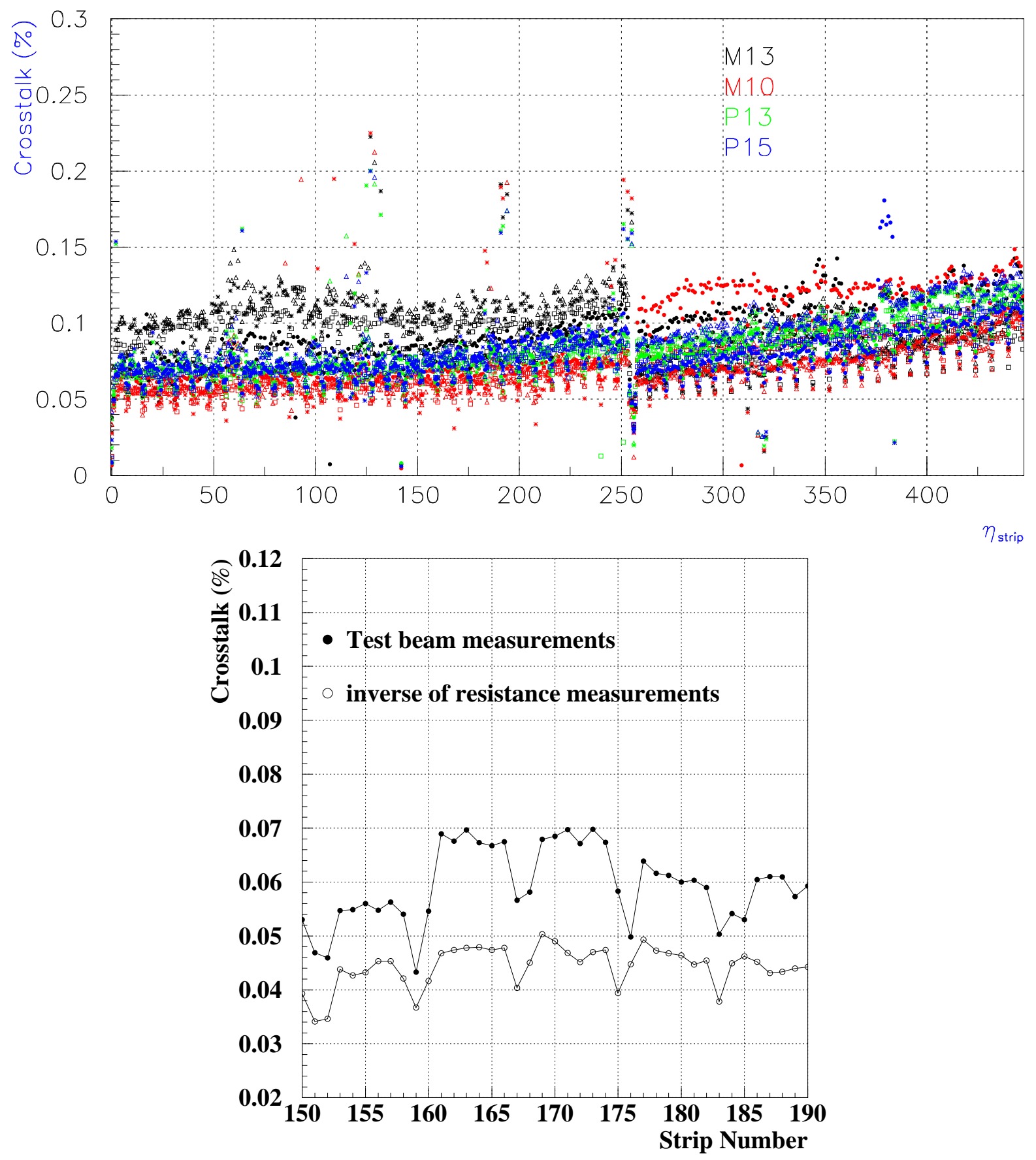

Figure 39: Upper: resistive crosstalk among adjacent strips for several production modules, cells at several $\phi$. Lower: comparison of the resistive crosstalk and the inverse of the resistor measured before stacking. The structure with an 8-fold periodicity is the same as in fig. 26 and comes from the resistor geometry in the electrode design. 

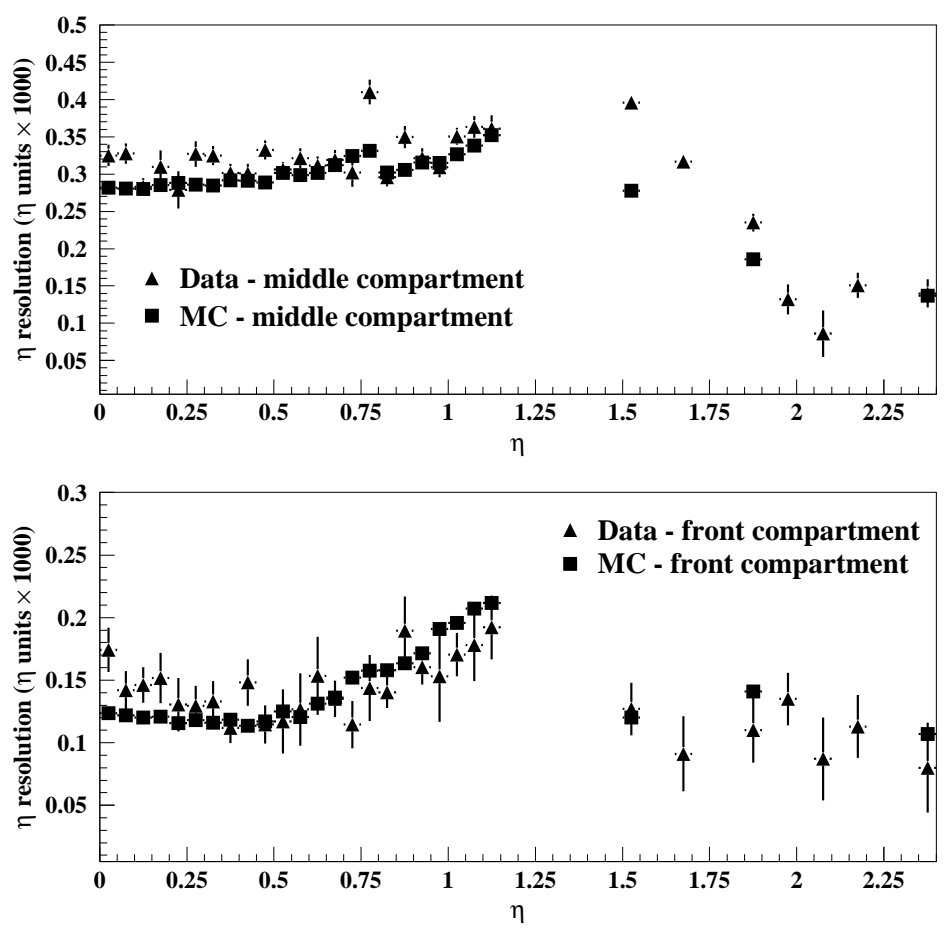

Figure 40: Position resolution versus $\eta$ in the middle (top) and front (lower) sections compared to the simulation.
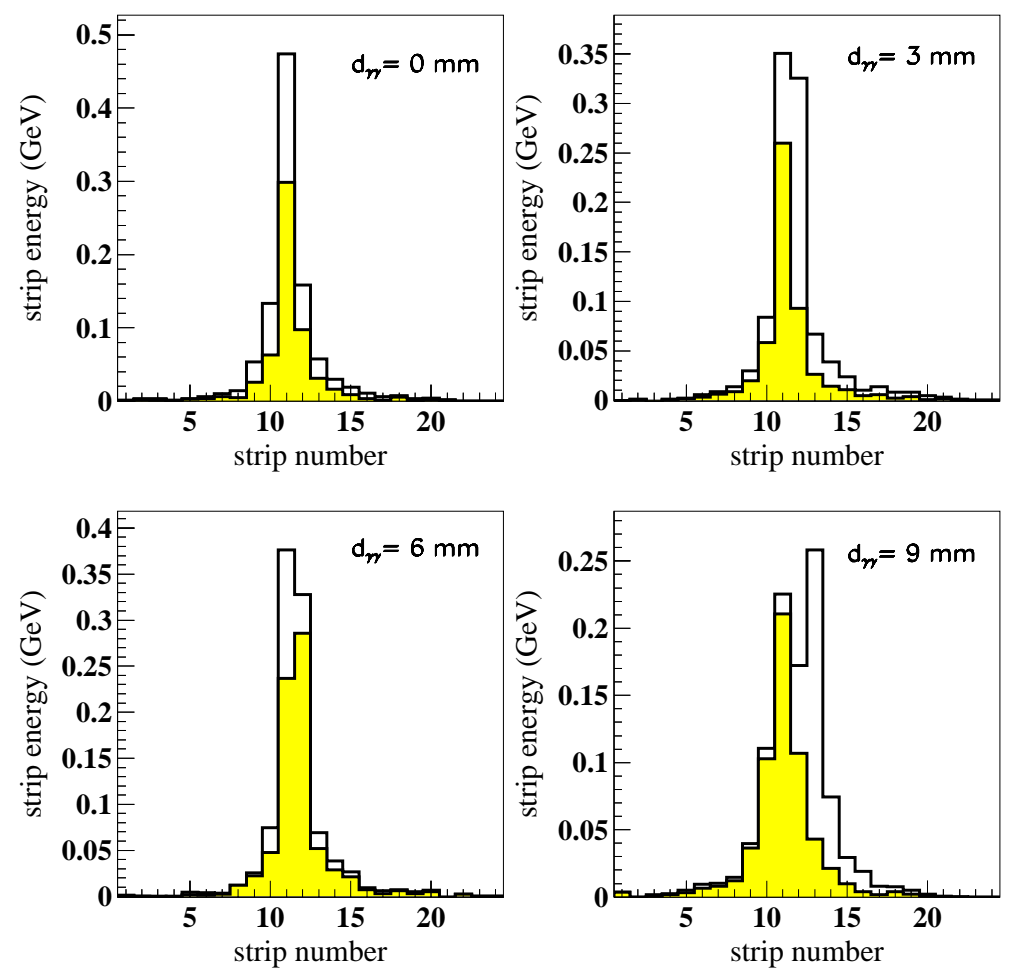

Figure 41: Shower profiles of constructed $\pi^{0}$ 's where the two photons are separated by 0 (upper left), 3 (upper right), 6 (lower left), $9 \mathrm{~mm}$ (lower right). In each case the shaded histogram corresponds to the most energetic of the two photons and the empty histogram contains the whole $\pi^{0}$ profile. 


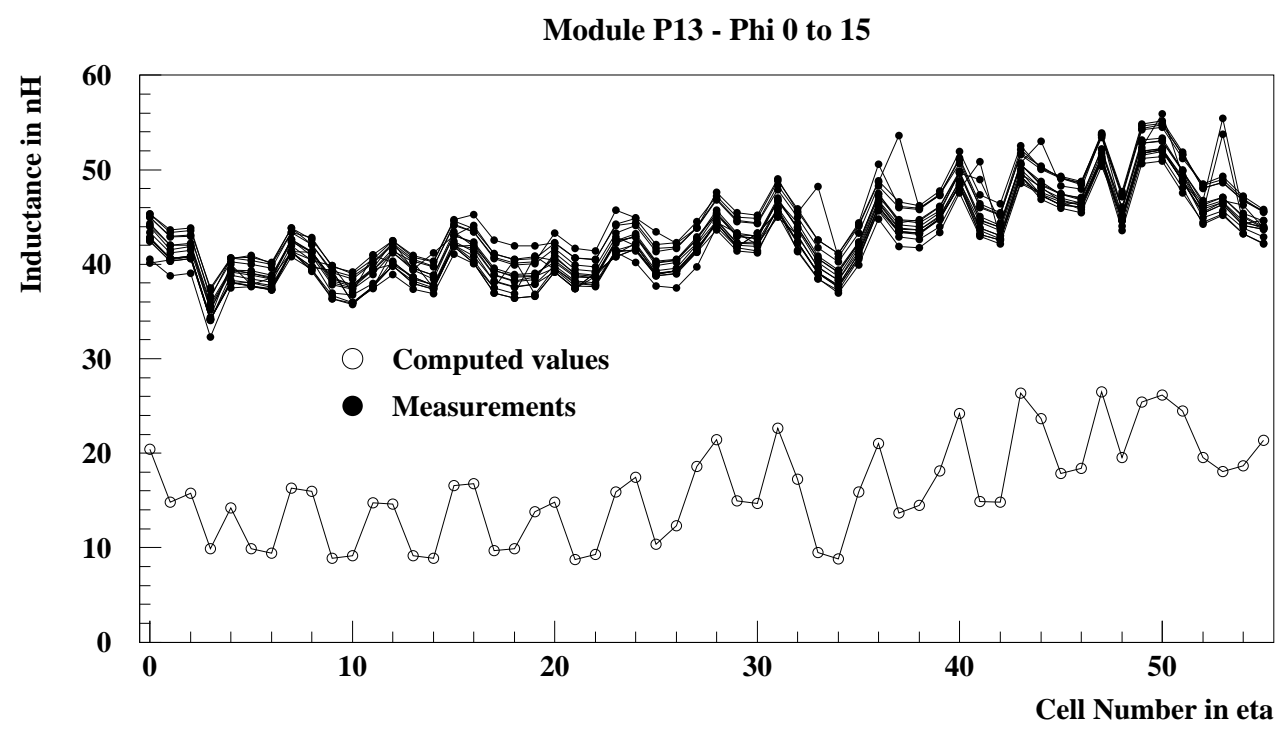

Figure 42: Inductance of middle cells along $\eta$ for several rows of $\phi$ for barrel module P13. Values are to be compared to the calculated ones (empty circles). The offset to the measured values (full circles) comes from the measurement setup.

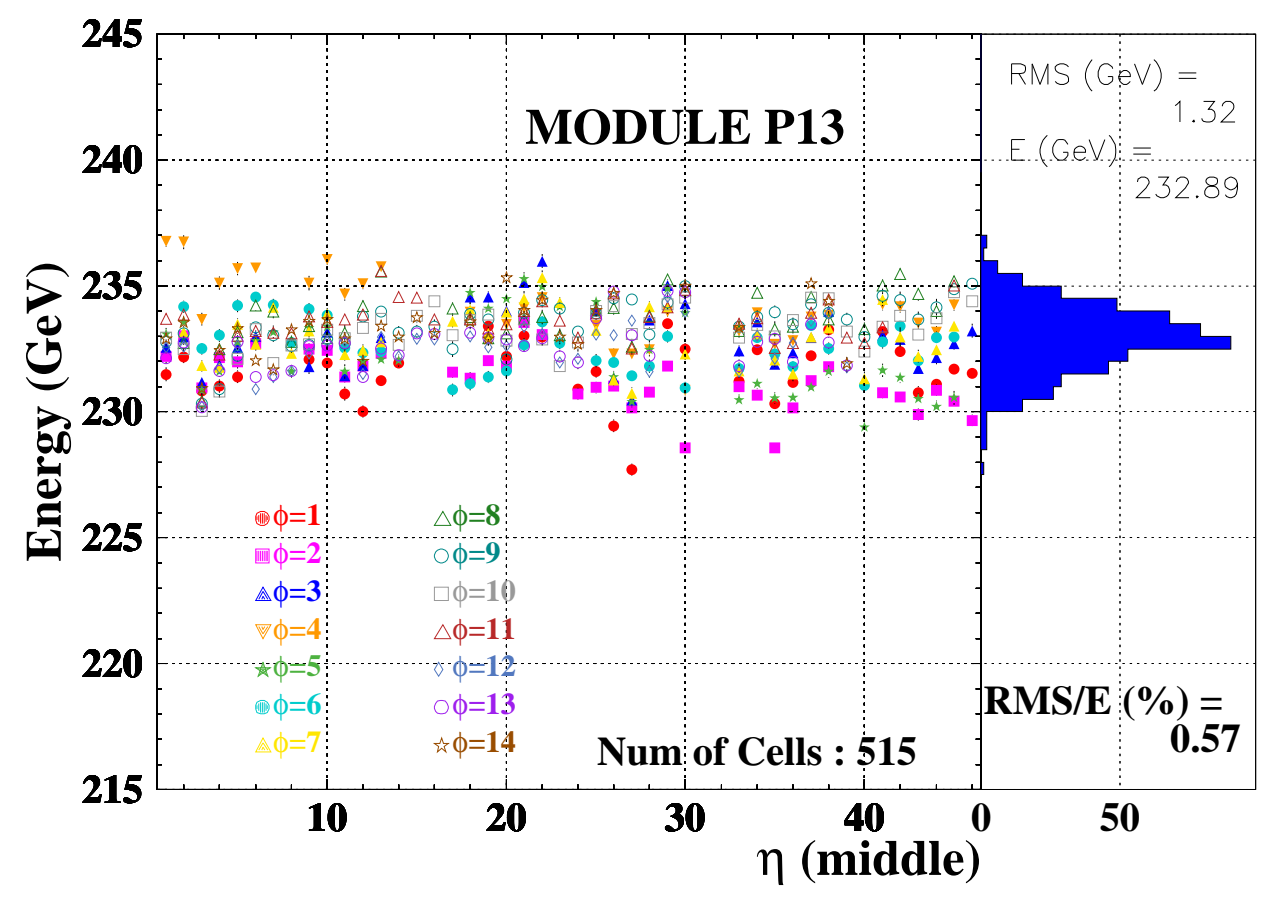

Figure 43: Energy response uniformity for a barrel module. 
steps of the circuit production needed to be mastered. A large effort was devoted to QA during production of flat circuits and to form, test and equip the electrodes in an accordion shape before stacking on the calorimeter modules.

Various results during the construction as well as with beam tests have shown that the electrodes and thus the EM LAr calorimeter perform to their design specification.

\section{Acknowledgements}

We appreciate the essential help by the technical staff of the participating laboratories. In particular we would like to thank P. Baudin, J.-F. Ballansat, J. Boniface, P. Brion, G. Broutinel, V. Clap, G. Dromby, L. Journet, P. Letournel, J. Orrit, D. Stanchina (LAPP, Annecy-le-Vieux), P. Lançon (CERN, Geneva), D. Tezier (CPPM, Marseille), R. Bertoni, G. Braga, B. Monticelli (INFN, Milano), O. Bohner, B. Debennerot, P. Favre, M. Fernandez, P. Imbert,R. Leray,V. Marsaud, M. Quentin (LAL, Orsay), M.-M. Cloarec, A. Commerçon, C. Goffin, P. Etienne, A. Guimard, P. Laloux, J.-M. Parraud, P. Repain, D. Steyaert (LPNHE, Paris). This project would not have been possible without the financial support from our funding agencies and additional support by CEA, DSM/DAPNIA, Saclay. For the long and difficult contractual negotiations we are indebted to D. Gregorio of the CERN Purchasing Service. We acknowledge also the support from a long list of companies providing different parts or services into this project. In particular we would like to thank Arisawa, Japan, who provided high quality laminates in non-commercial width and the management and staff of Cicorel SA of Boudry, Switzerland, where the series electrodes were produced for the long and cooperative collaboration in this adventure.

\section{References}

[1] ATLAS Collaboration, Liquid Argon Calorimeter Technical Design Report, CERN-LHCC-96-41.

[2] RD3 Collaboration, Nucl. Instrum. Methods A 309 (1991) 438; 321 (1992) 467; 325 (1993) 118; 389 (1997) 398; 364 (1995) 290.

[3] F.Djama, L. Hervas, C.P. Marin: Copper Polyimide Multilayer Electrodes for the End-Cap Electromagnetic Calorimeter, ATL-LARG-98-088; CERN, January 1998.

[4] W. Bonivento and D. Lacour, Acceptable values of the resistances on electrodes of the ATLAS e. m. Calorimeter, ATL-LARG-99-019; CERN, March 1999.

[5] W. Bonivento, Impact of resistor quality of the readout electrodes on e. m. calorimeter performance, ATL-LARG-97-081; CERN, September 1997.

[6] B. Aubert, S. Jezequel, C.P. Marin, Raw Material for Signal Electrodes, ATL-LARG-98-102; CERN, May 1998.

[7] M. L. Andrieux et al., Pollution of liquid argon after neutron irradiation measured at SARA: summary of raw data, ATL-LARG-98-105; CERN, May 1998.

[8] ATLAS Electromagnetic Liquid Argon Calorimeter Group, Nucl. Instrum. Methods A 500 (2003) 178.

[9] ATLAS Electromagnetic Liquid Argon Calorimeter Group, Nucl. Instrum. Methods A 500 (2003) 202.

[10] W. Bonivento, G. Costa, M. Mazzanti : The electrode test setup developed in Milano: experience with RD3 and ATLAS prototypes ATL-LARG-97-070; CERN, April 1997.

F. Astesan, B. Canton, D. Lacour, F. Rossel : Description and Performances of the Electrical Test Benches for Readout Electrodes of the ATLAS EM Calorimeter ATL-LARG-99-005; CERN, March 1999

[11] P. Dargent, F. Djama: Bending of the End-Cap electrodes, ATL-LARG-98-108; CERN, November 1998.

[12] Construction, assembly and first tests of the ATLAS electromagnetic calorimeter barrel, Nucl. Instrum. Methods A (in preparation.) 
[13] F. Hubaut, B. Laforge, D. Lacour, F. Orsini : Test Beam Measurement of the Crosstalk in the EM Barrel Module 0 ATL-LARG-2000-007; CERN, March 2000.

[14] F. Hubaut,Nucl. Instrum. Methods A 518 (2004) 31.

R. Sacco: Position Resolution of an Atlas Electromagnetic Calorimeter Module, ATL-LARG-2003-8; CERN, July 2003.

[15] R. Sacco : Performances of the ATLAS Electromagnetic Calorimeter Modules; in Proceedings of the 8th International Conference on Advanced Technology and Particle Physics, Como, 6-10 October 2003. 